\title{
Parafermions in hierarchical fractional quantum Hall states
}

\author{
Luiz H. Santos \\ Department of Physics, Emory University, Atlanta, Georgia 30322, USA
}

(Received 24 June 2019; accepted 10 January 2020; published 28 February 2020)

\begin{abstract}
Recent theoretical progress has been achieved in demonstrating the existence of non-Abelian parafermion zero modes in domain walls on interfaces between two-dimensional Abelian topological phases of matter. Motivated by this ongoing search for non-Abelian defects, we investigate the properties of gapped interfaces of hierarchical fractional quantum Hall states, in the lowest Landau level, characterized by the Hall conductance $\sigma_{x y}(m, p)=$ $\frac{p}{2 m p+1} \frac{e^{2}}{h}$, for integer numbers $(m, p)$ with $m, p \geqslant 1$. The case $m=1$ corresponds to the experimentally well established sequence of fractional quantum Hall states with $\sigma_{x y}=\frac{1}{3} \frac{e^{2}}{h}, \frac{2}{5} \frac{e^{2}}{h}, \frac{3}{7} \frac{e^{2}}{h}, \ldots$, which has been observed in many two-dimensional electron gases. Exploring the mechanism by which the $(m, p+1)$ hierarchical state is generated from the condensation of quasiparticles of the "parent" state $(m, p)$, we uncover a remarkably rich sequence of parafermions in hierarchical interfaces whose quantum dimension $d_{m, p}$ depends both upon the total quantum dimension $\mathcal{D}_{m, p}=\sqrt{2 m p+1}$ of the bulk Abelian phase, as well as on the parity of the "hierarchy level" $p$, which we associate with the $\mathbb{Z}_{2}$ stability of Majorana zero modes in one-dimensional topological superconductors. We show that these parafermions reside on domain walls separating segments of the interface where the low-energy modes are gapped by two distinct mechanisms: (1) a charge neutral backscattering process or (2) an interaction that breaks U(1) charge conservation symmetry and stabilizes a condensate whose charge depends on $p$. Remarkably, this charge condensate corresponds to clustering of quasiparticles of fractional charge $\frac{p}{2 m p+1} e$, allowing us to draw a correspondence between these fractionalized condensates and Read-Rezayi non-Abelian fractional quantum Hall cluster states.
\end{abstract}

DOI: 10.1103/PhysRevResearch.2.013232

\section{INTRODUCTION}

Topological phases of matter are promising systems to realize fault-tolerant quantum computation due to the longrange entanglement of the quantum many-body state [1]. Emergent quasiparticles in two-dimensional (2D) topological phases obeying fractional statistics are a potential resource for quantum information science, particularly so if the system hosts non-Abelian quasiparticles, which allow for the assembling of a degenerate ground-state manifold where quantum information can be stored and manipulated [2]. Non-Abelian phases have been theoretically investigated in a variety of contexts, from fractional quantum Hall (FQH) systems [3] to quantum spin liquids [4] and recent years have seen exciting experimental progress to detect signatures of non-Abelian quasiparticles $[5,6]$.

In the last two decades, it has been noticed that superconductivity is an important mechanism to stabilize emergent low-energy excitations with non-Abelian character. A well known example is Kitaev's one-dimensional (1D) $p$-wave superconductor supporting Majorana zero modes at the edges [7]. In this context, the edge of the finite system behaves as a

Published by the American Physical Society under the terms of the Creative Commons Attribution 4.0 International license. Further distribution of this work must maintain attribution to the author(s) and the published article's title, journal citation, and DOI. domain wall interpolating between a nontrivial superconductor and a charge neutral insulator, i.e., vacuum.

Recent theoretical breakthroughs in topological phases have demonstrated that emergent non-Abelian extrinsic defects can be stabilized as domain wall states in edges and interfaces or boundaries of 2D topological phases whose bulk quasiparticles obey solely Abelian statistics [8-17]. In certain cases previously considered, domain walls represent twist defects of an anyonic symmetry, which is a transformation that permutes the anyons without changing their fundamental statistical properties [4,13,14,18-21].

In the presence of such domain walls, the system can encode a nontrivial ground-state degeneracy. The zero modes in question, which constitute a generalization of Majorana fermions, are referred to as parafermions [22]. Parafermions have been introduced to describe phase transitions of 2D classical clock models with $\mathbb{Z}_{n}$ symmetry [23,24], and in recent years, there has been a renewed interest surrounding the relationship between parafermions and topological systems [25]. A system with $2 N$ parafermion zero modes furnishes a ground-state manifold with $d^{2 N}$ states [26], where $d$ represents the quantum dimension of the parafermion. Majorana fermions correspond to the special case $d=\sqrt{2}$, which occur as zero energy excitations in the edges of $1 \mathrm{D}$ topological superconductors [7] and at the vortex core of 2D chiral p-wave superconductors [27,28].

A 2D electron gas under external magnetic field provides a rich realization of Abelian phases of matter in the form of the FQH effect [29]. In addition to the Laughlin states [30], a plethora of $\mathrm{FQH}$ plateaus with quantized Hall conductance 
are observed upon changing the magnetic field or the electron density. A remarkable aspect of these topological phases, particularly when in the first Landau level, is their hierarchical organization [31-33] into a Jain sequence of incompressible states characterized by the quantized Hall conductance

$$
\sigma_{x y}(m, p)=\frac{e^{2}}{h} \frac{p}{2 m p+1},
$$

where $e$ is the electron charge, $h$ is the Planck constant, $m$ and $p$ are integer numbers greater or equal than one, which characterize the sequence of FQH states with filling fraction $v(m, p)=\frac{p}{2 m p+1}<1$. The index $p$ labels the "position" of the hierarchical state, whose primary state $(p=1$ and fixed value of $m$ ) is the Laughlin state with filling fraction $v(m, 1)=1 /(2 m+1)$. We shall refer to each of the hierarchical states above by a pair of integer numbers $(m, p)$. For instance, in the FQH plateaus of Hall conductance $\sigma_{x y} /\left(e^{2} / h\right)=$ $1 / 3,2 / 5,3 / 7,4 / 9, \ldots$, the condensation of quasiparticles of the primary Laughlin state with $\sigma_{x y} /\left(e^{2} / h\right)=1 / 3$ yields the first hierarchical state with $\sigma_{x y} /\left(e^{2} / h\right)=2 / 5$; which in turn gives rise to the second hierarchical state with $\sigma_{x y} /\left(e^{2} / h\right)=3 / 7$, and so on.

We note that, despite the large number of states accounted by Eq. (1.1), it represents a subset of the hierarchical states. In the composite fermion description [31,34], Jain states are understood to arise form the nucleation of an even number $(2 \mathrm{~m})$ of flux quanta per electron, leading to new degrees of freedom-composite fermions-filling an integer number $(p)$ of effective Landau levels. In this sense, the Jain sequence represents a transformation from a FQHE of electrons into a IQHE of composite fermions. Composite fermions can, on the other hand, partially populate a Landau level and form a FQH state of their own, leading to a new sequence of hierarchical states not accounted by Eq. (1.1). Examples of FQH plateaus that admit this description are the $v=4 / 11$ and $v=5 / 13 \mathrm{FQH}$ states, which have been observed [35]. Given the complexity of the problem, in this work we will concern ourselves with the description of parafermions in the hierarchical Jain sequence, noticing that the quest for non-Abelian defects in other hierarchical states merits its own separate investigation.

Even though the investigation of bulk topological properties of the hierarchy of Abelian FQH in the lowest Landau level has a long history [32,33,36-40], the relationship between the bulk anyon condensation and the properties of non-Abelian parafermion zero modes supported by these phases Abelian phases remains an open problem. The goal of this work is to address this problem, thus establishing a correspondence between the hierarchy of FQH states and the local interactions on their interfaces capable of stabilizing non-Abelian parafermion zero modes. We also note that, by means of a "folding transformation," the results obtained here for the nonchiral interfaces of hierarchical time-reversal symmetry breaking FQH states straightforwardly apply to the nonchiral edge states pertaining to the hierarchy of timereversal symmetric Abelian fractional topological insulators $[41,42]$.

In interfaces of Laughlin states with filling fraction $v=$ $\frac{1}{2 m+1}$ ( $m$ integer), a domain wall between a segment gapped by the charge neutral backscattering $H_{\mathrm{bs}}=\psi_{L}^{\dagger} \psi_{R}+$ H.c., where $\psi_{L}$ and $\psi_{R}$ are, respectively, the fermionic operators on the left and right edges of the interface-and another region gapped by the charge 2 condensate $H_{\text {pair }}=\psi_{L} \psi_{R}+$ H.c., supports a $\mathbb{Z}_{2(2 m+1)}$ parafermion with quantum dimension $d=$ $\sqrt{2(2 m+1)}$ [9-12]. The $m=0$ case corresponds to Majorana fermions on domain walls at the interface of the $v=1$ integer quantum Hall state, akin to the 1D p-wave superconductor [7]. The parafermion zero modes that occur for $m \geqslant 1$ then represent a fractionalization of the Majorana fermion. A noteworthy aspect of the quantum dimension of the parafermions $d=d_{1 D} \times d_{\text {bulk }}$, is that it receives a contribution from the fractionalized bulk Abelian state, $d_{\text {bulk }}=\sqrt{2 m+1}$, as well as a contribution $d_{1 D}=\sqrt{2}$ stemming from the $1 \mathrm{D}$ physics. This property of Laughlin-type interfaces raises some important questions regarding the remaining sequence of hierarchical states: (1) what is the nature of local interactions and domain walls responsible for parafermions? (2) Specifically, does a charge 2 pairing interaction play an important role for generic hierarchical states, in complete analogy to Laughlin states? (3) What is the interplay between bulk topology and 1D physics in determining the quantum dimension of parafermions?

In this work, we will address these questions using an effective Luttinger liquid theory to describe the low-energy modes of the homogeneous interface between hierarchical FQH states. The presence of chiral edge modes is a wellknown consequence of the topological order of the bulk hierarchical state described by the bulk Chern-Simons gauge theory [43]. In this effective edge/interface theory, local operators that open an energy gap are expressed as generalized sine-Gordon (local) operators, which satisfy a compatibility condition (or, null condition) to ensure a stable gapped fixed point [44]. Since the number of low-energy modes at the interface grows with the level of the hierarchy $p$, any attempt of a general understanding of the parafermion problem that applies to the entire hierarchical sequence would seem a hopeless question. Nevertheless, much to the contrary, we shall demonstrate that an general and comprehensive understanding of the universal properties of parafermions is possible because of the relationship established between states of the hierarchy via anyon condensation. Specifically, the anyon condensation mechanism translates into a hierarchy of $\mathrm{K}$ matrices describing the bulk anyons, which, in turn, will allow us to systematically study the local interactions that gap the homogeneous interfaces.

By an explicit analysis of the sine-Gordon gap opening operators at the homogeneous interface, we shall precisely identify local interactions that stabilize parafermion zero modes at domain walls separating gapped segments where charge conservation is preserved from other segments where the interactions break $U(1)$ charge conservation symmetry and give rise to a condensate of charge

$$
\mathcal{Q}_{m, p}= \begin{cases}2 p & p \in \text { odd } \\ p & p \in \text { even }\end{cases}
$$

which, notably, depends on the level $p$ of the hierarchy. In particular, it shows an even-odd effect as a function of $p$. Moreover, we observe that, in general, $\mathcal{Q}_{m, p}>2$, which departs from the Laughlin interface [9-12]. Thus, for $p \geqslant 2$, 


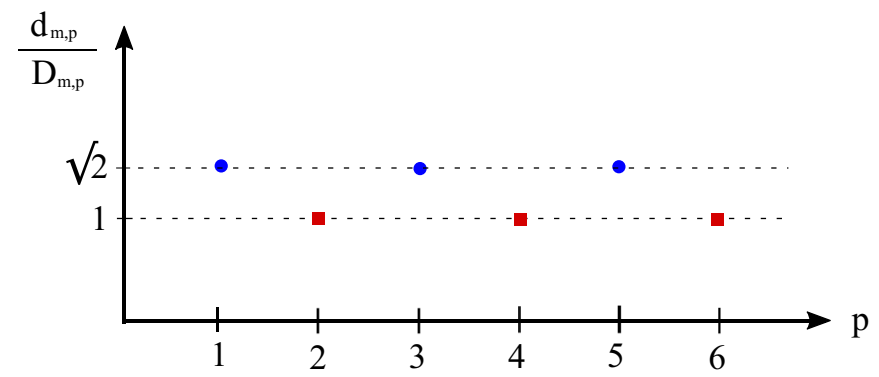

FIG. 1. Ratio of the parafermion quantum dimension $d_{m, p}$ to the total quantum dimension of the bulk Abelian hierarchical FQH phase $D_{m, p}$ plotted as a function of the hierarchy level $p$, for a fixed integer $m$. This plot captures the even-odd dependence given by Eq. (1.3), with blue circles and red squares representing, respectively, odd and even values of $p$.

we identify new forms of local interactions that require a clustering mechanism beyond the conventional BCS pairing [45]. Explicit form of these local interactions will be discussed in Secs. IV and V.

Our main result is summarized in Fig. 1. By studying the low-energy properties of the domain walls separating the U(1) symmetry preserving and broken regions of the interface, we will show that they support parafermion zero modes with quantum dimension

$$
d_{m, p}= \begin{cases}\sqrt{2} \mathcal{D}_{m, p} & p \in \text { odd } \\ \mathcal{D}_{m, p} & p \in \text { even }\end{cases}
$$

where $\mathcal{D}_{m, p}=\sqrt{2 m p+1}$ is the total quantum dimension of the bulk state, which is related to the topological entanglement entropy $[46,47]$ of the hierarchical state $(m, p)$ via $\gamma_{m, p}=$ $\ln \mathcal{D}_{m, p}$ and to the minimum quasihole charge $e_{m, p}^{*}=\mathcal{D}_{m, p}^{-2}$. We again notice the quantum dimension of the parafermions reflect an even-odd effect in terms of $p$, similarly to Eq. (1.2). Furthermore, the emergence of non-Abelian zero modes with quantum dimensions given by Eq. (1.3) suggest a different mechanism than that considered in Refs. [4,13,14,18,21], where the defects with non-Abelian character relate to twist defects of a symmetry of the anyon group of the Abelian state. For instance, in Sec. IV, we show that, in the first and second hierarchical states, twist defects of the charge conjugation anyonic symmetry behave as a Majorana fermions $(d=$ $\sqrt{2}$ ) and twist defects associated with a "layer permutation" anyonic symmetry [13] are trivial $(d=1)$, in contrast with Eq. (1.3).

Equations (1.2) and (1.3) embody a rich fractionalization phenomenon at the gapped interface. As shall be demonstrated here, a segment of the interface where the charge condensate (1.2) is realized is associated with the expectation value of an operator of charge $v_{m, p}=\frac{p}{2 m p+1}$ (recall $e=1$ unit), which shows that the charge $p$ operator (for $p$ even) is realized by a cluster of $(2 m p+1)$ quasiparticles. It turns out the appearance of $\mathbb{Z}_{2 m p+1}$ parafermions with quantum dimension $d_{m, p}=\sqrt{2 m p+1}$ is a direct consequence of this clustered state, as shall be explained later. In the odd $p$ case, the same type of charge condensate is formed, however, according to Eq. (1.3), domain walls support $\mathbb{Z}_{2(2 m p+1)} \cong$
$\mathbb{Z}_{2} \oplus \mathbb{Z}_{2 m p+1}$ parafermions with quantum dimension $d_{m, p}=$ $\sqrt{2} \times \sqrt{2 m p+1}$, where the extra $\mathbb{Z}_{2}$ structure is reminiscent of Majorana zero models in 1D topological superconductors.

We argue that this even-odd effect is a manifestation of the $\mathbb{Z}_{2}$ classification of $1 \mathrm{D}$ topological superconductors, where the integer index $p$ plays the role of the number of stacked copies of 1D topological superconductors. The interpretation of this result is natural in the hydrodynamical Abelian Chern-Simons theory of the hierarchical FQH states, where the universal information of the hierarchical states $(m, p)$ is represented by a square integer valued $K$ matrix of dimension $p$ [36-40]. Under a suitable $\operatorname{SL}(p, \mathbb{Z})$ transformation, the $K$ matrix can be interpreted as a $p$-layer FQH system, which reduces to an integer quantum Hall system of $p$ filled Landau when $m=0$. This interpretation of the Chern-Simons hydrodynamical theory will enable contact with the $\mathbb{Z}_{2}$ classification of 1D topological superconductors and support the validity of Eq. (1.3), which will be explicitly derived in Sec. V.

The anyon cluster state realized at the homogeneous interface of hierarchical FQH states bears a remarkable resemblance with the Read-Rezayi non-Abelian states where the ground state is build from clusters of $k$ electrons which yield a gapped bulk with non-Abelian quasiparticles and an edge that supports a chiral neutral $\mathbb{Z}_{k}$ parafermion mode [48]. The case $k=2$ corresponds to the Moore-Read paired state [3] where electrons (or composite fermions) form a paired state whose neutral sector is described by an effective chiral p-wave superconductor [27]. Reference [15] has shown that the $v=2 / 3$ FQH coupled to a superconductor can support $\mathbb{Z}_{3}$ parafermion zero modes on domain walls. Quite remarkably, hybridization of the $\mathbb{Z}_{3}$ parafermions modes throughout the bulk can give rise to a non-Abelian phase with properties similar to the $\mathbb{Z}_{3}$ Read-Rezayi FQH states that supports non-Abelian anyons capable of realizing universal quantum computation $[15,49]$. By the same token, and given the generality of the results established here, we expect that the deconfinement of parafermion zero modes realized in the hierarchy of Abelian $\mathrm{FQH}$ states to give rise to a rich class of $2 \mathrm{D}$ non-Abelian phases, thus unveiling fresh connections between families of Abelian and non-Abelian phases.

This paper is organized as follows. In Sec. II, we give an overview of the 2D hydrodynamical Chern-Simons theory for the bulk hierarchical Abelian FQH states [36-40]. The topological information about the hierarchical Abelian state $(m, p)$ is encoded by the $K_{m, p}$ matrix and charge vector $q_{m, p}$. An fundamental point of this discussion is that the hierarchy of Abelian states, related by anyon condensation, establishes a useful mapping between $K$ matrices of the elements of the hierarchy: $K_{m, 1} \rightarrow K_{m, 2} \rightarrow \cdots \rightarrow K_{m, p-1} \rightarrow K_{m, p} \rightarrow \ldots$, with a corresponding mapping for the charge vectors. This recursive form of the $K$ matrix and charge vector will be explored in order to establish the properties expressed in Eqs. (1.3) and (1.2). In Sec. III, we provide a general discussion of the properties of domain walls and parafermion zero modes on the interface of hierarchical states, where we shall consider two sets of local interactions, one that preserves and one that breaks $U(1)$ charge conservation. An important take home message of this general discussion is that the quantum 
dimension of parafermions can be efficiently calculated for any hierarchical $(m, p) \mathrm{FQH}$ state, despite the fact that the number of edge modes scales with the hierarchy level $p$. Then, in Sec. IV, we apply this formalism to the Laughlin primary states $(p=1)$ and the first three hierarchical states $p=2,3$, and 4. (In the case $m=1$, these represent the FQH states with filling fractions $2 / 5,3 / 7$, and 4/9.) This explicit analysis will be crucial in pointing to the general hierarchy of parafermions, which will be worked out in Sec. V. Finally, in Sec. VI, we shall summarize and discuss our results, as well as present perspectives for future directions.

\section{OVERVIEW OF THE HIERARCHY OF ABELIAN FOH STATES}

The stability of the sequence of incompressible hierarchical FQH states characterized by quantized $\sigma_{x y}(m, p)=$ $\frac{e^{2}}{h} \frac{p}{2 p m+1}$ can be understood in terms of the effective nucleation of an even number $2 m$ of flux quanta per electronimplemented by a Chern-Simons gauge field [34]—giving rise to composite fermions [31,50] which, at mean-field level, occupy an integral number $p$ of filled effective Landau levels.

An alternative description to the composite fermion approach employs a Chern-Simons hydrodynamical theory to capture the universal properties of the hierarchical FQH states [36-40]. This hydrodynamical approach explains the sequence of Abelian FQH states in terms of sequential anyon condensations. Given the $(2+1)$ dimensionality of the problem, electron and quasiparticle conserved currents each can be parametrized by a U(1) gauge field. The condensation of quasiparticles in a given plateau state labeled by $(m, p)$ then gives rise to the hierarchical state $(m, p+1)$, whose effective theory contains an additional Chern-Simons field. The resulting effective theory of the $(m, p)$ hierarchical state then corresponds to an Abelian Chern-Simons theory that depends upon $p$ flavors of gauge fields, where the Aharonov-Bohm phases associated with exchange of gauge fluxes are encoded in the integral square and symmetric $K$ matrix of dimension $p$, which characterizes the Abelian topological order of the hierarchical FQH state $(m, p)$.

In addition to the Abelian statistics of bulk quasiparticles, the hydrodynamical Chern-Simons theory yields direct information about the low-energy properties of the edge states, which form a chiral Luttinger liquid [43]. The enlargement of the dimension of the $K$ matrix as a function of the hierarchical parameter $p$ signals the increase in the number of chiral edge modes. Therefore, when considering local interactions among the modes of such an interface, it is seen that opening of an energy gap is achieved by generalized sine-Gordon local operators whose forms are constrained by the $K$ matrix of the bulk state, which in turn provides a potent link between local operators at the interface and the bulk topological order.

The above-mentioned correspondence between the bulk phase and local edge operators will be explored to establish a correspondence between the hierarchical Abelian states and the parafermions zero modes in their interfaces. Given the importance of this formalism, in Sec. II A, we review essential elements of the hydrodynamical Chern-Simons theory of 2D hierarchical FQH states [36-40] leading to the recursive form of the $K$ matrix and charge vector and, later in Sec. II B, we make contact the chiral 1D Luttinger liquid theory governing the low-energy physics of the edge states [43].

\section{A. Abelian Chern-Simons theory of the hierarchical FQH states}

Throughout the rest of the paper, we work in units where $e=\hbar=1$, unless when when we present formulas for the Hall conductance where the fundamental constants will be explicitly displayed.

Let us begin with Laughlin states at filling fraction $v_{m, 1}=$ $1 /(2 m+1)$, which are the primary states $(m, 1)$ of the hierarchical sequence. Their effective low-energy theory is captured by the 2D bulk Chern-Simons Lagrangian

$$
L_{m, 1}^{2 D}=-\frac{2 m+1}{4 \pi} \varepsilon^{\alpha \beta \gamma} a_{\alpha}^{1} \partial_{\beta} a_{\gamma}^{1}+\frac{1}{2 \pi} \varepsilon^{\alpha \beta \gamma} A_{\alpha} \partial_{\beta} a_{\gamma}^{1},
$$

where $a_{\mu}^{1}$ is a dynamical Chern-Simons gauge field, $A_{\mu}$ is the external electromagnetic field, Greek indices account for space-time coordinates $\{0,1,2\}=\{t, x, y\}$ and the conserved electric current is $J^{\alpha}=\frac{1}{2 \pi} \varepsilon^{\alpha \beta \gamma} \partial_{\beta} a_{\gamma}^{1}$. Furthermore, here and throughout, repeated indices are summed over. Integrating out the Chern-Simons gauge field $a_{\mu}^{1}$ yields the electromagnetic response

$$
L_{m, 1}^{2 D, \text { response }}=\frac{1}{4 \pi} \frac{1}{2 m+1} \varepsilon^{\alpha \beta \gamma} A_{\alpha} \partial_{\beta} A_{\gamma}
$$

that encodes the Hall conductance $\sigma_{x y}(m, 1)=\frac{e^{2}}{h} \frac{1}{2 m+1}$.

Expressing the quasiparticle conserved current by $j_{2}^{\alpha}=$ $\frac{1}{2 \pi} \varepsilon^{\alpha \beta \gamma} \partial_{\beta} a_{\gamma}^{2}$, expressed in terms of the gauge field $a_{\mu}^{2}$, the effective theory of the first hierarchical state $(m, 2)$ is given by

$$
\begin{aligned}
L_{m, 2}^{2 D}= & -\frac{2 m+1}{4 \pi} \varepsilon^{\alpha \beta \gamma} a_{\alpha}^{1} \partial_{\beta} a_{\gamma}^{1}+\frac{1}{2 \pi} \varepsilon^{\alpha \beta \gamma} A_{\alpha} \partial_{\beta} a_{\gamma}^{1} \\
& +\frac{1}{4 \pi} \varepsilon^{\alpha \beta \gamma} a_{\alpha}^{1} \partial_{\beta} a_{\gamma}^{2}+\frac{1}{4 \pi} \varepsilon^{\alpha \beta \gamma} a_{\alpha}^{2} \partial_{\beta} a_{\gamma}^{1} \\
& -\frac{2}{4 \pi} \varepsilon^{\alpha \beta \gamma} a_{\alpha}^{2} \partial_{\beta} a_{\gamma}^{2},
\end{aligned}
$$

where the first two terms of the second line of Eq. (2.3) cone from the minimal coupling $j_{2}^{\mu} a_{\mu}^{1}$ and the last term captures the property that, in the mean-field state, the density of quasiparticles $j^{0}$ satisfies $j^{0}=\frac{1}{2} \frac{\nabla \times a^{1}}{2 \pi}$, implying they condense forming a bosonic Laughlin state with filling $1 / 2$.

By introducing the Chern-Simons doublet $a_{\mu}^{T}=\left(a_{\mu}^{1}, a_{\mu}^{2}\right)$, Eq. (2.3) reads

$$
\begin{aligned}
& L_{m, 2}^{2 D}=-\frac{1}{4 \pi} \varepsilon^{\alpha \beta \gamma} a_{\alpha}^{T} K_{m, 2} \partial_{\beta} a_{\gamma}+q^{T} \frac{1}{2 \pi} \varepsilon^{\alpha \beta \gamma} A_{\alpha} \partial_{\beta} a_{\gamma}, \\
& K_{m, 2}=\left(\begin{array}{cc}
2 m+1 & -1 \\
-1 & 2
\end{array}\right), \quad q_{m, 2}=(1,0)^{T} .
\end{aligned}
$$

Then, integrating out the Chern-Simons fields yields the electromagnetic response

$$
L_{m, 2}^{2 D, \text { response }}=\frac{1}{4 \pi} \frac{2}{4 m+1} \varepsilon^{\alpha \beta \gamma} A_{\alpha} \partial_{\beta} A_{\gamma},
$$

which encodes the Hall conductance $\sigma_{x y}(m, 2)=\frac{e^{2}}{h} \frac{2}{4 m+1}$ of the first hierarchical state. 
Carrying out these previous steps sequentially generates the hydrodynamical Chern-Simons theory of the hierarchy of Abelian FQH states [36-40]

$$
\begin{aligned}
& L_{m, p+1}^{2 D}=-\frac{1}{4 \pi} \varepsilon^{\alpha \beta \gamma} a_{\alpha}^{T} K_{m, p+1} \partial_{\beta} a_{\gamma}+q^{T} \frac{1}{2 \pi} \varepsilon^{\alpha \beta \gamma} A_{\alpha} \partial_{\beta} a_{\gamma}, \\
& K_{m, p+1}=\left(\begin{array}{ccccc} 
& & & & 0 \\
& & K_{m, p} & & \vdots \\
& & & & 0 \\
0 & \ldots & 0 & -1 & 2
\end{array}\right), \\
& q_{m, p+1}=\left(q_{m, p} 0\right)^{T} \text {. }
\end{aligned}
$$

The physical mechanism by which the $(m, p+1)$ daughter state is generated from the condensation of anyons of the $(m, p)$ parent state is mathematically manifested in the recursive form of the $K$ matrix (2.6b) and the charge vector (2.6c). Moreover,

$$
\operatorname{det}\left(K_{m, p}\right)=2 m p+1,
$$

gives the torus ground-state degeneracy of the $\mathrm{FQH}$ state and measures the total quantum dimension of the Abelian topological phase

$$
\mathcal{D}_{m, p}=\sqrt{\left|\operatorname{det}\left(K_{m, p}\right)\right|}=\sqrt{2 m p+1} .
$$

Finally, the Hall conductance of the $(m, p)$ state, obtained from integrating out the Chern-Simons, is given by

$$
\sigma_{x y}(m, p)=\frac{e^{2}}{h} q_{m, p}^{T} K_{m, p}^{-1} q_{m, p}=\frac{e^{2}}{h} \frac{p}{2 m p+1} .
$$

It turns out that, for purpose of studying the properties of parafermion zero modes at the interface of hierarchical states, the recursive structure embodied in the $K$ matrix and charge vector in Eq. (2.6) will play a central role as shall be discussed in Secs. IV and V.

An important consideration is that the topological field theory (2.6) is only defined up to an $\operatorname{SL}(p, \mathbb{Z})$ transformation $a_{\mu} \rightarrow\left(W^{T}\right)^{-1} a_{\mu}, K_{m, p} \rightarrow W K_{m, p} W^{T}$ and $q_{m, p} \rightarrow W q_{m, p}$, which represents a relabeling of the quasiparticles that leaves their statistics unchanged. This freedom can be explored to represent the hierarchical $\mathrm{FQH}$ state in the alternative basis [38]

$$
\begin{aligned}
& \tilde{K}_{m, p}=W_{p} K_{m, p} W_{p}^{T}=\left(\begin{array}{cccccc}
2 m+1 & 2 m & 2 m & 2 m & \ldots & 2 m \\
2 m & 2 m+1 & 2 m & 2 m & \ldots & 2 m \\
2 m & 2 m & 2 m+1 & 2 m & \ldots & 2 m \\
\vdots & & & & & \\
2 m & 2 m & 2 m & \ldots & 2 m & 2 m+1
\end{array}\right), \\
& \tilde{q}_{m, p}=W_{p} q_{m, p}=(1,1, \ldots, 1)^{T},
\end{aligned}
$$

where

$$
W_{p}=\left(\begin{array}{cccccc}
1 & 0 & 0 & 0 & \ldots & 0 \\
1 & 1 & 0 & 0 & \ldots & 0 \\
1 & 1 & 1 & 0 & \ldots & 0 \\
\vdots & & & & & \\
1 & 1 & 1 & 1 & \ldots & 1
\end{array}\right) \in \operatorname{SL}(p, \mathbb{Z})
$$

The $K$ matrix (2.8a) has the following appealing interpretation: the diagonal odd integers $2 m+1$ alone represent a system of $p$ layers of Laughlin $v=1 /(2 m+1)$ states. The even off-diagonal factors of $2 m$, on the other hand, represent bosonic correlations among the $p$ fermionic layers. Notice the charge vector (2.8b) denotes that the each layer carries unit charge under the external electromagnetic field. It will prove useful to explore both representations (2.6) and (2.8) when describing the properties of parafermions.

\section{B. Luttinger liquid theory of the hierarchical edge states}

According to the bulk-boundary correspondence, the bulk topological phase given by Eq. (2.6a) supports a chiral edge
Luttinger liquid [43]

$$
\begin{aligned}
\mathcal{L}_{m, p}^{R}= & -\frac{1}{4 \pi} \partial_{t} \Phi_{R}^{T} \cdot K_{m, p} \cdot \partial_{x} \Phi_{R}-\frac{1}{4 \pi} \partial_{x} \Phi_{R}^{T} \cdot V_{m, p}^{R} \cdot \partial_{x} \Phi_{R} \\
& +\frac{1}{2 \pi} q_{m, p}^{T} \varepsilon^{\alpha \beta} A_{\alpha} \partial_{\beta} \Phi_{R},
\end{aligned}
$$

where $V_{m, p}^{R}$ is a positive-definite matrix ensuring a bounded edge spectrum. Furthermore, since all the $p$ eigenvalues of $K_{m, p}$ are positive, the edge contains $p$ right-moving modes described by the fields $\Phi_{R}$, which are depicted at the bottom part of the interface shown in Fig. 2(a). The top part of the interface supports left-moving modes that are described a similar Lagrangian as in Eq. (2.9), albeit with an appropriate sign change of the $K$ matrix that reflects the opposite orientation of the edge modes. Therefore the Luttinger liquid theory of the interface reads

$$
\begin{aligned}
\mathcal{L}_{m, p}= & -\frac{1}{4 \pi} \partial_{t} \Phi^{T} \cdot \mathcal{K}_{m, p} \cdot \partial_{x} \Phi-\frac{1}{4 \pi} \partial_{x} \Phi^{T} \cdot \mathcal{V}_{m, p} \cdot \partial_{x} \Phi \\
& +\frac{1}{2 \pi} Q_{m, p}^{T} \varepsilon^{\alpha \beta} A_{\alpha} \partial_{\beta} \Phi,
\end{aligned}
$$

where

$$
\Phi=\left(\begin{array}{c}
\Phi_{R} \\
\Phi_{L}
\end{array}\right)
$$


(a)

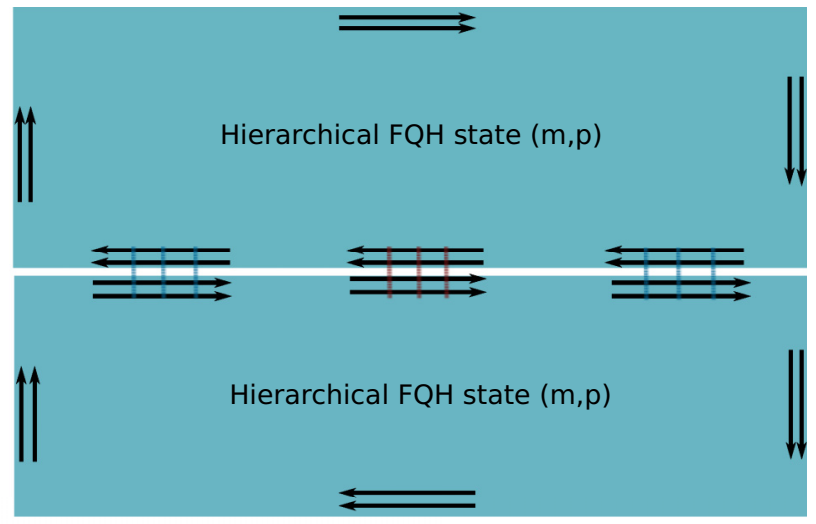

(b)

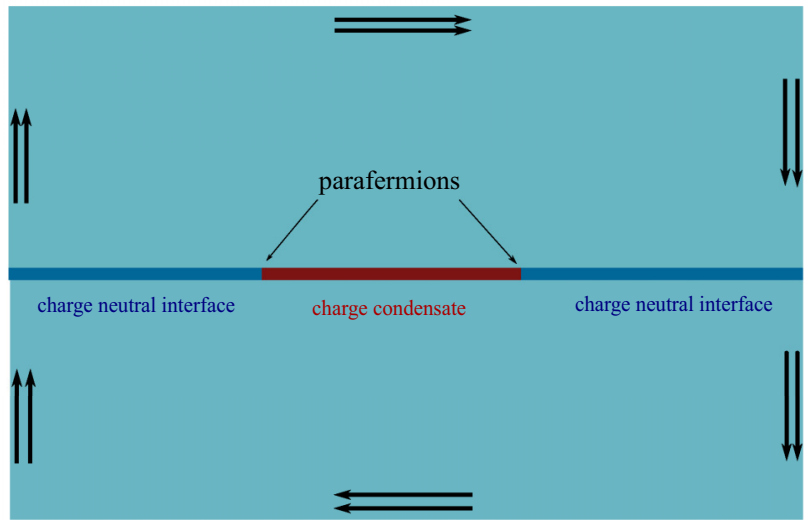

FIG. 2. (a) Top and bottom panels show the same hierarchical state, each one supporting a number $p$ of chiral edge modes, which form a nonchiral interface. Blue and red dotted lines represent charge conserving and $U(1)$ symmetry breaking operators that open an energy gap at the interface. (b) Blue and red lines at the interface represent segments gapped by competing mass terms, with domain walls supporting parafermion zero modes.

$\Phi_{R}$ and $\Phi_{L}$ are, respectively, the right- and left-moving $p$ tuplet of bosonic edge fields,

$$
Q_{m, p}^{T}=\left(q_{m, p}^{T},-q_{m, p}^{T}\right)=(1,0, \ldots, 0,-1,0, \ldots, 0)
$$

is the charge vector and

$$
\mathcal{K}_{m, p}=\left(\begin{array}{cc}
K_{m, p} & 0 \\
0 & -K_{m, p}
\end{array}\right)
$$

is the $K$ matrix of the interface. The equal-time commutation relations of the edge fields reads

$$
\left[\partial_{x} \Phi_{a}(x), \Phi_{b}\left(x^{\prime}\right)\right]=2 \pi i\left(\mathcal{K}_{m, p}^{-1}\right)_{a b} \delta\left(x-x^{\prime}\right) .
$$

Gapping the $p$ pairs of counter propagating modes at the interface is achieved with a set of $p$ commuting sine-Gordon local interactions

$$
U\left[\Lambda_{i}\right]=\cos \left(\Lambda_{i}^{T} \mathcal{K}_{m, p} \Phi\right), \quad i=1, \ldots, p,
$$

where $\Lambda_{i}$ are $2 p$-component integer vectors representing correlated backscattering processes between right- and leftmoving local quasiparticles at the interface. Furthermore, owing to the nontrivial commutation relations, Eq. (2.11), satisfied by the edge fields, the integer vectors $\Lambda_{i}$ are required to satisfy the null condition [44]

$$
\Lambda_{i}^{T} \mathcal{K}_{m, p} \Lambda_{j}=0, \quad i, j=1, \ldots, p
$$

in order for the local interactions (2.12) to form a compatible set of mutually bosonic operators. Moreover, the integer U(1) charge of the operator $U\left[\Lambda_{i}\right]$ is

$$
\mathcal{Q}\left[\Lambda_{i}\right]=\Lambda_{i}^{T} Q_{m, p} .
$$

Clearly, integer vectors continue to satisfy Eq. (2.13) upon rescaling by an integer greater than one. Then with the respect to the Luttinger liquid fixed point, Eq. (2.10), these rescaled null vectors describe local operators with larger scaling dimensions, which are then less relevant at low energy and can be disregarded. Therefore, in the remaining of this paper, we shall only focus our attention on null vectors that are primitive [42]. As shown in that work, a single integer vector is primitive when the greatest common divisor of its entries is 1 . A set of integer vectors is primitive if and only if the greatest common divisor of the set of minors of the $p \times 2 p$ integer matrix $\mathcal{M}[\{\Lambda\}]$ is 1 , where $\mathcal{M}[\{\Lambda\}]$ is the integer matrix whose rows are formed by the null vectors $\Lambda_{i}$.

In general, the low-energy modes of the interface can become gapped due to distinct types of local interactions, each one associated with a primitive null set $\left\{\Lambda_{i}\right\}$ satisfying Eq. (2.13). As the number $p$ of counter-propagating modes at the interface grows (i.e., as one moves "deeper" into the hierarchical sequence), one expects a corresponding increase in the number of gapping channels of the interface, as a consequence of more available backscattering channels amongst the counter-propagating modes. As such, the investigation of the low-energy properties of interfaces of hierarchical $\mathrm{FQH}$ states poses a very rich physics problem. In the following discussion, we shall concentrate on certain classes of local interactions leading to gapped interfaces, whose properties will be described in generality in Sec. III and, more specifically, in Secs. IV and V.

\section{DOMAIN WALLS IN HIERARCHICAL INTERFACES: GENERAL PROPERTIES}

We now discuss the properties of domain walls and parafermion zero modes associated with hierarchical interfaces. One of the central points of this section is the ansatz (3.4) that describes the $U(1)$ symmetry broken interactions at the interface and which will permit us to determine, efficiently, the quantum dimension of the parafermions localized on the domain walls for an interface that holds $p$ counter propagating modes.

As described in Sec. II B, the number of chiral edge modes grows with the hierarchy index $p$, which increases the number of gap opening channels. In this context, addressing all possible forms of gapped interfaces seems a formidable task, which is beyond the scope of this work. Instead, we shall focus on a specific class of local interactions, which will be shown to stabilize parafermion zero modes on domain walls along the interface. We shall consider two types of gapped interfaces. The first one is formed by charge neutral backscattering, while the second one breaks charge conservation. Our focus is then on the low-energy properties of domain walls separating charge conserving and the nonconserving gapped segments. 
We note that superconducting pairing correlations have been recently induced in integer quantum Hall edges [51,52], which represents a promising step to create superconductor/FQH heterostructures.

The homogeneous interface described by Eq. (2.10) admits local charge neutral backscattering that gap the interface and heal the bulk states, as represented by the blue segments in Fig. 2(b). This interface, which allows Abelian anyons to hop across and propagate as bona fide deconfined bulk quasiparticles, is created by the backscattering terms

$$
U\left[\Lambda_{i}^{(0)}\right]=\cos \left(\Lambda_{i}^{(0)} \mathcal{K}_{m, p} \Phi\right)
$$

where

$$
\Lambda_{i}^{(0)}=\left(\begin{array}{c}
e_{i} \\
e_{i}
\end{array}\right), \quad e_{i}=(0, \ldots, 0, \underbrace{1}_{i}, 0, \ldots, 0)^{T}
$$

for $i=1, \ldots, p$ is a set of integer vectors. Charge conservation obeyed by the interactions (3.1a) follows from

$$
\mathcal{Q}\left[\Lambda_{i}^{(0)}\right]=\Lambda_{i}^{(0)} Q_{m, p}=\left(\begin{array}{ll}
e_{i}^{T} & e_{i}^{T}
\end{array}\right)\left(\begin{array}{c}
q_{m, p} \\
-q_{m, p}
\end{array}\right)=0,
$$

and the null condition of the integer vectors (3.1b)

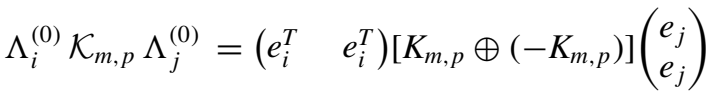

$$
\begin{aligned}
& =e_{i}^{T} K_{m, p} e_{j}-e_{i}^{T} K_{m, p} e_{j}=0
\end{aligned}
$$

is verified $\forall i, j=1, \ldots, p$.

We now consider another set of local interactions

$$
U\left[\Lambda_{i}\right]=\cos \left(\Lambda_{i} \mathcal{K}_{m, p} \Phi\right)
$$

that break charge conservation and depend upon the integer vectors

$$
\Lambda_{i}= \begin{cases}\Lambda_{1} \neq \Lambda_{1}^{(0)} & \text { and } \Lambda_{1} Q_{m, p} \neq 0 \\ \Lambda_{i}^{(0)} & i=2, \ldots, p\end{cases}
$$

for $i=1, \ldots, p$.

The interaction $U\left[\Lambda_{1}\right]$ breaks charge conservation, while the $U\left[\Lambda_{i \neq 1}\right]$ conserve charge. Despite its simple form, this ansatz will be shown to embody a nontrivial charge condensate that gaps the interface and stabilizes parafermions on domain walls between segments of the interface gapped by the interactions (3.4a) from those segments gapped by the charge neutral interactions (3.1a). Furthermore it permits an analytical understanding of the mechanism behind the formation of domain wall parafermions. Since the subset of $p-1$ null vectors $\left\{\Lambda_{i}=\Lambda_{i}^{(0)}, i=2, \ldots, p\right\}$ in Eq. (3.4b) satisfies the null condition, Eq. (2.13) reduces to $p$ independent equations that can be solved exactly, as we shall demonstrate in the following Secs. IV and V.

In order to determine the quantum dimension of the parafermion zero modes, we consider a series of domain walls at the interface that separate segments $S_{0}=\cup_{i}\left(x_{2 i}+\right.$ $\left.\varepsilon, x_{2 i+1}-\varepsilon\right)$ gapped by the interactions (3.1a) from the segments $S=\cup_{i}\left(x_{2 i-1}+\varepsilon, x_{2 i}-\varepsilon\right)$ gapped by the interactions (3.4a), where $\varepsilon=0^{+}$is a positive regulator for the domain walls. In the strong coupling limit, the ground state is obtained by locking the sine-Gordon terms (3.1a) and (3.4a) to their minima on the respective segments $S^{(0)}$ and $S$. The groundstate degeneracy can be obtained by constructing a set of operators with support on these gapped segments

$$
\begin{aligned}
& \Gamma_{2 i-1,2 i}=\exp \left(\frac{i}{N_{m, p}} \int_{x_{2 i-1}-\varepsilon}^{x_{2 i}+\varepsilon} d x \Lambda_{1}^{(0)} \mathcal{K}_{m, p} \partial_{x} \Phi\right) \\
& \Gamma_{2 i, 2 i+1}=\exp \left(\frac{i}{N_{m, p}} \int_{x_{2 i}-\varepsilon}^{x_{2 i+1}+\varepsilon} d x \Lambda_{1} \mathcal{K}_{m, p} \partial_{x} \Phi\right)
\end{aligned}
$$

where

$$
N_{m, p}=\Lambda_{1}^{(0)} \mathcal{K}_{m, p} \Lambda_{1} \in \mathbb{Z}^{*} .
$$

It follows from the commutation relations (2.11) that operators defined in Eqs. (3.5a) and (3.5b) commute with the Hamiltonian along the interface and satisfy the algebra

$$
\begin{aligned}
\Gamma_{2 i-1,2 i} \Gamma_{2 j, 2 j+1} & =e^{i \frac{2 \pi}{N_{m, p}}\left(\delta_{i, j}-\delta_{i-1, j}\right)} \Gamma_{2 j, 2 j+1} \Gamma_{2 i-1,2 i}, \\
\Gamma_{2 k-1,2 k}^{N_{m, p}} & =\Gamma_{2 k, 2 k+1}^{N_{m, p}}=1 .
\end{aligned}
$$

The dimension of the minimum representation of this algebra clearly corresponds to the ground-state degeneracy. $\Gamma_{2 i, 2 i+1}$ act as raising or lowering operator to its neighbors $\Gamma_{2 i-1,2 i}$ and $\Gamma_{2 i+1,2 i+2}$, as $\mathbb{Z}_{N_{m, p}}$ clock operators. For a configuration with $2 n_{d w}$ domain walls, Eq. (3.6) conveys the ground-state degeneracy $\left|N_{m, p}\right|^{n_{d w}}$ and the quantum dimension of the parafermion

$$
d_{m, p}=\sqrt{\left|N_{m, p}\right|}
$$

Therefore the quantum dimension of the parafermions depends upon a single integer given by Eq. (3.5c).

Finally, the ground-state degeneracy stems from the existence of parafermion zero modes on the domain walls

$$
\begin{aligned}
\alpha_{2 i} & =e^{\frac{i}{N_{m, p}}\left[\Lambda_{1} \mathcal{K}_{m, p} \Phi\left(x_{2 i}-\varepsilon\right)+\Lambda_{1}^{(0)} \mathcal{K}_{m, p} \Phi\left(x_{2 i}+\varepsilon\right)\right],} \\
\alpha_{2 i+1} & =e^{\frac{i}{N_{m, p}}\left[\Lambda_{1}^{(0)} \mathcal{K}_{m, p} \Phi\left(x_{2 i+1}-\varepsilon\right)+\Lambda_{1} \mathcal{K}_{m, p} \Phi\left(x_{2 i+1}+\varepsilon\right)\right]},
\end{aligned}
$$

which satisfy the $\mathbb{Z}_{N_{m, p}}$ parafermion algebra

$$
\alpha_{i} \alpha_{j}=e^{i \frac{2 \pi}{N_{m, p}} \operatorname{sgn}(i-j)} \alpha_{j} \alpha_{i}
$$

and are related to the operators in Eq. (3.5) by

$$
\alpha_{2 i}^{\dagger} \alpha_{2 i+1} \sim \Gamma_{2 i+1,2 i},
$$

with similar relations holding for the other segments on the interface. This establishes that the bilinear terms constructed out of the parafermion operators commute with the Hamiltonian at the interface.

In the following section, we shall impose the null and primitive conditions to the interactions given by the ansatz (3.4) and use it to obtain the local U(1) symmetry broken interactions and parafermion zero modes for the first few hierarchical states. (For completeness we shall also revisit the primary Laughlin state studied in Refs. [9-12].) The analysis of these explicit cases will point to the general formulation valid for all hierarchical states, which we shall present in Sec. V. 


\section{HIERARCHY OF PARAFERMIONS: EXAMPLES}

In this section, we apply the formalism introduced in Sec. III from the primary Laughlin states up until the third hierarchical state.

\section{A. Primary Laughlin state $(m, 1)$}

The Laughlin state with filling fraction $v_{m, 1}=\frac{1}{2 m+1}=\frac{1}{3}$, $\frac{1}{5}, \ldots$ is described by a one-component quantum fluid with $K_{m, 1}=2 m+1$ and $q_{m, 1}=1$. The interface Luttinger liquid theory, Eq. (2.10), has

$$
\mathcal{K}_{m, 1}=\left(\begin{array}{cc}
2 m+1 & 0 \\
0 & -(2 m+1)
\end{array}\right), \quad Q_{m, 1}=(1,-1)^{T},
$$

with electron operators given by $\psi_{L / R}=e^{i(2 m+1) \phi_{L / R}}$, where $\phi_{L / R}$ are the chiral boson fields at the interface.

The charge neutral backscattering (3.1) for the Laughlin interface reads

$$
\begin{aligned}
\Lambda_{1}^{(0)} & =(1,1), \\
U\left[\Lambda_{1}^{(0)}\right] & =\frac{\lambda_{1}}{2}\left(\psi_{L}^{\dagger} \psi_{R}+\text { H.c. }\right) \\
& =\lambda_{1} \cos \left[(2 m+1)\left(\phi_{R}-\phi_{L}\right)\right] .
\end{aligned}
$$

Alternatively, the interface can be gapped via charge 2 electron pairing.

$$
\begin{aligned}
\Lambda_{1} & =(1,-1), \\
U\left[\Lambda_{1}\right] & =\frac{\lambda_{1}^{\prime}}{2}\left(\psi_{L} \psi_{R}+\text { H.c. }\right) \\
& =\lambda_{1}^{\prime} \cos \left[(2 m+1)\left(\phi_{R}+\phi_{L}\right)\right] .
\end{aligned}
$$

Then, according to Eq. (3.5c),

$$
N_{m, 1}=\Lambda_{1}^{(0)} \mathcal{K}_{m, 1} \Lambda_{1}=2(2 m+1)
$$

establishes $\mathbb{Z}_{2(2 m+1)} \cong \mathbb{Z}_{2} \oplus \mathbb{Z}_{2 m+1}$ parafermions

$$
\alpha_{i} \alpha_{j}=e^{i \frac{2 \pi}{2(2 m+1)} \operatorname{sgn}(i-j)} \alpha_{j} \alpha_{i}
$$

with quantum dimension

$$
d_{m, 1}=\sqrt{2} \times \sqrt{2 m+1}=\sqrt{2} \times \mathcal{D}_{m, 1},
$$

which are localized on the domain walls between regions gapped by the charge neutral local backscattering (4.3) and regions gapped by the charge 2 paring (4.5) [9-12]. Notice that the quantum dimension of the parafermion shows a contribution from the bulk topological order through the total quantum dimension $\mathcal{D}_{m, 1}=\sqrt{2 m+1}$ of the Laughlin state, as well as a contribution $\sqrt{2}$ reminiscent of Majorana zero modes in a 1D topological superconductor [7]. Because of this $1 \mathrm{D}$ effect, even in the absence of deconfined bulk anyons, which corresponds to the $v=1 \mathrm{IQH}$ state where $m=0$, there is one Majorana zero mode localized on each domain wall of the IQH interface.

The presence of $\mathbb{Z}_{2(2 m+1)}$ parafermions on the domain walls, as seen by Eq. (3.8), manifests that the charge $1 / \mathrm{m}$ operator

$$
\left\langle\mathcal{O}_{\frac{1}{2 m+1}}\right\rangle=\left\langle e^{i \frac{(2 m+1)\left(\phi_{R}+\phi_{L}\right)}{2(2 m+1)}}\right\rangle=\left\langle e^{i \frac{\left(\phi_{R}+\phi_{L}\right)}{2}}\right\rangle \neq 0
$$

acquires a nonzero expectation on the segments of the interface that are gapped by the interaction (4.5).

\section{B. First hierarchical state $(m, 2)$}

The interface of the first hierarchical state with filling fraction $v_{m, 2}=\frac{2}{4 m+1}=\frac{2}{5}, \frac{2}{9}, \ldots$ contains two pairs of counterpropagating fields $\Phi^{T}=\left(\phi_{1}^{R}, \phi_{2}^{R}, \phi_{1}^{L}, \phi_{2}^{L}\right)$. The Luttinger liquid Lagrangian of the interface, Eq. (2.10), has

$$
\begin{aligned}
& \mathcal{K}_{m, 2}=K_{m, 2} \oplus\left(-K_{m, 2}\right), \quad Q_{m, 2}^{T}=\left(q_{m, 2}^{T},-q_{m, 2}^{T}\right), \\
& K_{m, 2}=\left(\begin{array}{cc}
2 m+1 & -1 \\
-1 & 2
\end{array}\right), \quad q_{m, 2}^{T}=(1,0) \text {. }
\end{aligned}
$$

where we adopt the hierarchical representation $(2.6 \mathrm{~b})$ for the $K$ matrix of the bulk state.

According to Eq. (3.1), the pair of local interactions

$$
\begin{aligned}
U\left[\Lambda_{1}^{(0)}\right] & =\frac{\lambda_{1}}{2}\left(\psi_{1 L}^{\dagger} \psi_{1 R}+\text { H.c. }\right) \\
& =\lambda_{1} \cos \left[(2 m+1)\left(\phi_{1 R}-\phi_{1 L}\right)-\left(\phi_{2 R}-\phi_{2 L}\right)\right]
\end{aligned}
$$

and

$$
\begin{aligned}
U\left[\Lambda_{2}^{(0)}\right] & =\frac{\lambda_{2}}{2}\left(\psi_{2 L}^{\dagger} \psi_{2 R}+\text { H.c. }\right) \\
& =\lambda_{2} \cos \left[-\left(\phi_{1 R}-\phi_{1 L}\right)+2\left(\phi_{2 R}-\phi_{2 L}\right)\right],
\end{aligned}
$$

associated with the null vectors

$$
\Lambda_{1}^{(0)}=(1,0,1,0), \quad \Lambda_{2}^{(0)}=(0,1,0,1),
$$

gap the interface without breaking charge conservation. The local operators at the interface correspond to $\psi_{a, R / L}=$ $e^{i \sum_{b}\left(K_{R / L}\right)_{a b} \phi_{b, R / L}}$, for $a=1,2$. Equation (4.11) represents local charge neutral backscattering that localizes the interface lowenergy modes.

We now seek the null vectors and corresponding charge nonconserving interactions that gap the modes of the interface. Following Eq. (3.4), we consider

$$
\Lambda_{1}=\left(x_{1}, y_{1}, x_{2}, y_{2}\right), \quad \Lambda_{2}=(0,1,0,1),
$$

where $x_{1}, x_{2}, y_{1}, y_{2}$ are integers. Notice that the interaction $U\left[\Lambda_{2}\right]=\cos \left(\Lambda_{2} \mathcal{K}_{m, 2} \Phi\right)$ is charge neutral, while $U\left[\Lambda_{1}\right]$ is an operator of charge $\mathcal{Q}\left[\Lambda_{1}\right]=\Lambda_{1} Q_{m, 2}=x_{1}-x_{2}$, and we consider $x_{1} \neq x_{2}$ in what follows.

The null condition satisfied by the integer vectors (4.12) reads

$$
\begin{aligned}
\Lambda_{1} \mathcal{K}_{m, 2} \Lambda_{1}= & (2 m+1)\left(x_{1}^{2}-x_{2}^{2}\right) \\
& +2\left(y_{1}^{2}-y_{2}^{2}-x_{1} y_{1}+x_{2} y_{2}\right)=0, \\
\Lambda_{1} \mathcal{K}_{m, 2} \Lambda_{2}= & -x_{1}+x_{2}+2 y_{1}-2 y_{2}=0 .
\end{aligned}
$$

Solving for $x_{1}$ in Eq. (4.13b) and substituting into Eq. (4.13a) gives

$$
\left(y_{1}-y_{2}\right)\left(x_{2}+y_{1}-y_{2}\right)=0 .
$$

Whereas $y_{1}=y_{2}=y$ and $x_{1}=x_{2}=x$ solves the null conditions, it corresponds to a charge neutral null vector $\Lambda_{1}=x \Lambda_{1}^{(0)}+y \Lambda_{2}^{(0)}$ that is a linear combination of those in Eq. (4.11c), in which case the integer vectors (4.11c) 


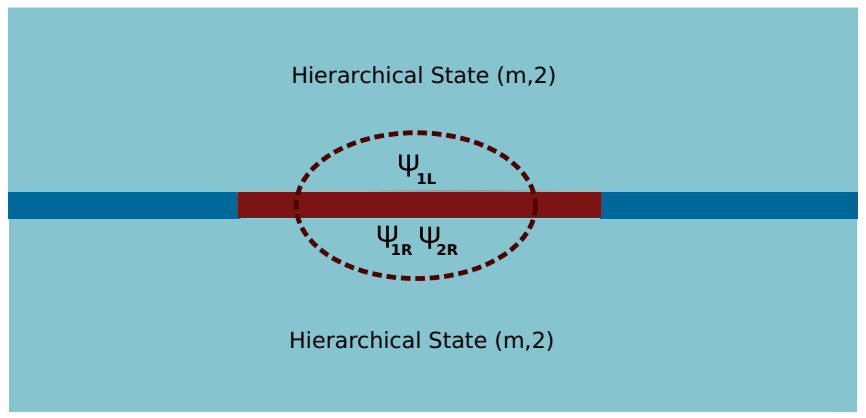

FIG. 3. Interface between the first hierarchical FQH state $(m, 2)$. The red segment represents the interface gapped by the charge 2 interaction (4.16), where $\psi_{1 R}$ and $\psi_{1 L}$ are both charge 1 fermionic operators, and $\psi_{2 R}$ accounts for a charge zero operator with bosonic self-statistics.

and (4.12) represent the same type of gapped interface. A nontrivial solution, however, corresponds to $y_{1}-y_{2}=t$, and $x_{1}=-x_{2}=t$ for $t \neq 0$, such that $\Lambda_{1}=\left(t, t+y_{2},-t, y_{2}\right)$ for $y_{2} \in \mathbb{Z}$. Moreover, the minors of $\mathcal{M}\left[\left\{\Lambda_{1}, \Lambda_{2}\right\}\right]$, are given by $\{t, 0, t, t, t,-t\}$, such that the primitive condition requires $t= \pm 1$. Finally, setting $t=1$ and $y_{2}=0$ yields

$$
\Lambda_{1}=(1,1,-1,0) \text {, }
$$

and the gap opening interaction

$$
\begin{aligned}
U\left[\Lambda_{1}\right] & =\frac{u}{2}\left(\psi_{1 R} \psi_{2 R} \psi_{1 L}+\text { H.c. }\right) \\
& =u \cos \left(\Lambda_{1} \mathcal{K}_{m, 2} \Phi\right) \\
& =u \cos \left(2 m \phi_{1}^{R}+\phi_{2}^{R}+(1+2 m) \phi_{1}^{L}-\phi_{2}^{L}\right) .
\end{aligned}
$$

This interaction represents a charge 2 condensate, where $\psi_{1 R}$ and $\psi_{1 L}$ are both charge 1 fermionic operators, and $\psi_{2 R}$ accounts for a charge zero operator with bosonic self-statistics. See Fig. 3.

According to Eq. (3.5c),

$$
N_{m, 2}=\Lambda_{1}^{(0)} \mathcal{K}_{m, 2} \Lambda_{1}=4 m+1
$$

shows the presence of $\mathbb{Z}_{4 m+1}$ parafermions

$$
\alpha_{i} \alpha_{j}=e^{i \frac{2 \pi}{4 m+1} \operatorname{sgn}(i-j)} \alpha_{j} \alpha_{i}
$$

with quantum dimension

$$
d_{m, 2}=\sqrt{4 m+1}=\mathcal{D}_{m, 2},
$$

which are localized on the domains of the interface. This result shows that the quantum dimension of the parafermion is a direct manifestation of bulk topological order of the first hierarchical state $(m, 2)$ through its total quantum dimension $\mathcal{D}_{m, 2}=\sqrt{4 m+1}$

As an application of our analysis, the homogeneous interface of $v=2 / 5 \mathrm{FQH}$ states (where $m=1$ and $p=2$ ) supports $\mathbb{Z}_{5}$ parafermions. In this case, parafermion operators are constructed from operators that create fractional charge $2 / 5$ and charge zero quasiparticle pairs on each side to the domain walls. Interestingly, the charge 2 condensate results from the coalescence of a quintuplet of quasiparticles of charge $2 / 5$.
The generalization of the $v=2 / 5$ state to other first hierarchical states with arbitrary values of $m$ shows that the $\mathbb{Z}_{4 m+1}$ parafermion results from the formation of a $(4 m+1)$-tuplet of charge $2 /(4 m+1)$ fractional quasiparticles giving rise to a charge 2 condensate. This, in turn, is a manifestation of the nonzero expectation value of the charge $\frac{2}{4 m+1}$ operator

$$
\left\langle\mathcal{O}_{\frac{2}{4 m+1}}\right\rangle \equiv\left\langle e^{i \frac{\Lambda_{1} \mathcal{K}_{m, 2} \Phi}{4 m+1}}\right\rangle \neq 0
$$

on the segments of gapped by the interaction (4.16).

Compared with the primary Laughlin states discussed in Sec. IV A, the quantum dimension of the parafermions in the first hierarchical state does not manifest a $\sqrt{2}$ contribution expected for the 1D topological superconductor. To shed light on this result, we make use of the representation of the first hierarchical state (2.8)

$$
\begin{aligned}
& \tilde{K}_{m, 2}=W_{2} K_{m, 2} W_{2}^{T}=\left(\begin{array}{cc}
2 m+1 & 2 m \\
2 m & 2 m+1
\end{array}\right), \\
& \tilde{q}_{m, 2}=(1,1),
\end{aligned}
$$

where the first hierarchical state can be thought of as a coupled FQH bilayer, with each layer carrying U(1) charge $q=1$. Therefore the degrees of freedom at the interface constitute twopairs of counter-propagating fermion modes-twice the number of degrees of freedom in the interface of the primary Laughlin states. Our analysis then shows that adding an extra pair of counter-propagating modes renders the Majorana zero mode unstable, which is an indication of the $\mathbb{Z}_{2}$ stability of Majorana fermions in 1D topological superconductors.

We now draw an important comparison between the parafermion zero modes discussed in our set up and the theory of extrinsic defects associated with anyonic symmetries of the Abelian phase. In Ref. [13], the $\mathbb{Z}_{2}$ twisted defects associated with the layer permutation of the Abelian phase characterized by the $K$ matrix

$$
K_{m, \ell}=\left(\begin{array}{cc}
m & \ell \\
\ell & m
\end{array}\right)
$$

where studied and it was shown that domain walls separating two distinct gapping charge conserving gap terms support parafermions with quantum dimension $d_{\mathbb{Z}_{2}}=\sqrt{|m-\ell|}$. Comparing (4.21) and (4.22) shows that that $\mathbb{Z}_{2}$ twist defect of the first hierarchical state is trivial, since $d_{\mathbb{Z}_{2}}=1$. Furthermore, it can be demonstrated [53] that the twist defects associated with charge conjugation anyonic symmetry correspond Majorana fermions, but not the $\mathbb{Z}_{4 m+1}$ parafermions discussed here.

These preliminary findings regarding the primary and first hierarchical states point to the existence of an outstanding even-odd effect that ties the stability of a Majorana zero mode to the parity of the hierarchical index $p$, as shown in Fig. 1. In the next two sections, we shall validate this even-odd effect by explicitly showing that the parafermions of second hierarchical state $(p=3)$ possess a $\sqrt{2}$ contribution to their quantum dimension, similar to the primary states $(p=1)$ in Sec. IV A; on the other hand, parafermions of third hierarchical state $(p=4)$ repeat the same behavior as those of the first hierarchical state $(p=2)$. 


\section{Second hierarchical state $(m, 3)$}

The low-energy modes of the interface of the second hierarchical state are described in terms of the fields $\Phi^{T}=\left(\phi_{1}^{R}, \phi_{2}^{R}, \phi_{3}^{R}, \phi_{1}^{L}, \phi_{2}^{L}, \phi_{3}^{L}\right)$ and the Lagrangian (2.10) has

$$
\begin{aligned}
& \mathcal{K}_{m, 3}=K_{m, 3} \oplus\left(-K_{m, 3}\right), \quad Q_{m, 3}^{T}=\left(q_{m, 3}^{T},-q_{m, 3}^{T}\right), \\
& K_{m, 3}=\left(\begin{array}{ccc}
2 m+1 & -1 & 0 \\
-1 & 2 & -1 \\
0 & -1 & 2
\end{array}\right), \quad q_{m, 3}^{T}=(1,0,0),
\end{aligned}
$$

where we adopt the hierarchical representation (2.6b).

The local charge neutral interactions (3.1) take the form

$$
\begin{aligned}
U\left[\Lambda_{i}^{(0)}\right] & =\left(\lambda_{i} / 2\right)\left(\psi_{i R}^{\dagger} \psi_{i L}+\text { H.c. }\right) \\
& =\cos \left(\Lambda_{i}^{(0)} \mathcal{K}_{m, 3} \Phi\right), \quad \text { for } i=1,2,3,
\end{aligned}
$$

associated with the null vectors

$$
\begin{aligned}
& \Lambda_{1}^{(0)}=(1,0,0,1,0,0), \\
& \Lambda_{2}^{(0)}=(0,1,0,0,1,0), \\
& \Lambda_{3}^{(0)}=(0,0,1,0,0,1) .
\end{aligned}
$$

Following Eq. (3.4), we now consider another set of null vectors

$$
\begin{aligned}
& \Lambda_{1}=\left(x_{1}, y_{1}, z_{1}, x_{2}, y_{2}, z_{2}\right), \\
& \Lambda_{2}=\Lambda_{2}^{(0)}=(0,1,0,0,1,0), \\
& \Lambda_{3}=\Lambda_{3}^{(0)}=(0,0,1,0,0,1),
\end{aligned}
$$

(where $x_{1}, \ldots, z_{2}$ are integers) corresponding to the local interactions

$$
U\left[\Lambda_{i}\right]=\cos \left(\Lambda_{i} \mathcal{K}_{m, 3} \Phi\right) \quad i=1,2,3,
$$

where $U\left[\Lambda_{2}\right]$ and $U\left[\Lambda_{3}\right]$ are charge neutral and $U\left[\Lambda_{1}\right]$ carries charge $x_{1}-x_{2} \neq 0$. Imposing the null condition results in three equations

$$
\begin{aligned}
\Lambda_{1} \mathcal{K}_{m, 3} \Lambda_{1}= & (2 m+1)\left(x_{1}^{2}-x_{2}^{2}\right)-2 x_{1} y_{1}, \\
& +2 x_{2} y_{2}+2\left(y_{1}^{2}-y_{1} z_{1}-y_{2}^{2}+y_{2} z_{2}+z_{1}^{2}-z_{2}^{2}\right) \\
= & 0 \\
\Lambda_{1} \mathcal{K}_{m, 3} \Lambda_{2}= & -x_{1}+x_{2}+2\left(y_{1}-y_{2}\right)-z_{1}+z_{2}=0 \\
\Lambda_{1} \mathcal{K}_{m, 3} \Lambda_{3}= & -y_{1}+y_{2}+2\left(z_{1}-z_{2}\right)=0 .
\end{aligned}
$$

Equations (4.28b) and (4.28c) result in

$$
z_{1}-z_{2}=\frac{x_{1}-x_{2}}{3}=\frac{y_{1}-y_{2}}{2}
$$

which gives

$$
\left[2 x_{2}+3\left(z_{1}-z_{2}\right)\right]\left(z_{1}-z_{2}\right)=0
$$

upon substitution onto Eq. (4.28a).

A nontrivial solution of Eqs. (4.29) and (4.30) yields the null vector $\Lambda_{1}=\left(3 t, y_{2}+4 t, z_{2}+2 t,-3 t, y_{2}, z_{2}\right)$ for $t \neq 0$ and $y_{2}, z_{2} \in \mathbb{Z}$. Furthermore, the nonzero minors of $\mathcal{M}\left[\left\{\Lambda_{1}, \Lambda_{2}, \Lambda_{3}\right\}\right]$ belong in the set $\{ \pm 2 t, \pm 3 t, \pm 4 t\}$ from which the primitive condition follows for $t= \pm 1$. Finally, setting $t=1, y_{2}=-2$, and $z_{2}=-1$, gives the null vector

$$
\Lambda_{1}=(3,2,1,-3,-2,-1),
$$

and the corresponding local interaction

$$
U\left[\Lambda_{1}\right]=\cos \left(\Lambda_{1} \mathcal{K}_{m, 3} \Phi\right)=\cos \left[(6 m+1)\left(\phi_{1}^{R}+\phi_{1}^{L}\right)\right] .
$$

It follows from Eqs. (4.31) and (3.5c) that

$$
N_{m, 3}=\Lambda_{1}^{(0)} \mathcal{K}_{m, 3} \Lambda_{1}=2(6 m+1),
$$

which establishes the presence of $\mathbb{Z}_{2(6 m+1)} \cong \mathbb{Z}_{2} \oplus \mathbb{Z}_{6 m+1}$ parafermions

$$
\alpha_{i} \alpha_{j}=e^{i \frac{2 \pi}{2(6 m+1)} \operatorname{sgn}(i-j)} \alpha_{j} \alpha_{i}
$$

with quantum dimension

$$
d_{m, 3}=\sqrt{2} \times \sqrt{6 m+1}=\sqrt{2} \times \mathcal{D}_{m, 3} .
$$

This explicit calculation, therefore, confirms that the structure of the parafermions in the second hierarchical state is similar to that observed in the primary Laughlin states discussed in Sec. IV A, with the quantum dimension of the parafermion being a manifestation of both the bulk Abelian order and the nontrivial 1D superconductor. Nevertheless, an important distinction emerges in this case, for the interaction (4.32) represents a condensate of charge $\mathcal{Q}\left[\Lambda_{1}\right]=Q_{m, 3} \Lambda_{1}=6$. The stability of parafermions, as seen in Eq. (3.8), is captured by the expectation value of the charge $\frac{3}{6 m+1}$ operator

$$
\left\langle\mathcal{O}_{\frac{3}{6 m+1}}\right\rangle \equiv\left\langle e^{i \frac{\Lambda_{1}^{(0)} \mathcal{K}_{m, 3} \Lambda_{1}}{2(6 m+1)}}\right\rangle=\left\langle e^{i \frac{\left(\phi_{1}^{L}+\phi_{1}^{R}\right)}{2}}\right\rangle \neq 0,
$$

on the segments of the interface gapped by this interaction. As an example, the interface between two FQH states at filling fraction $v=3 / 7$ (corresponding to $m=1, p=3$ ), can give rise to $\mathbb{Z}_{14} \cong \mathbb{Z}_{2} \oplus \mathbb{Z}_{7}$ parafermions along the domain walls described here. The charge 6 condensate, in this case, is formed by condensation of fractional charge $3 / 7$.

It is instructive to seek an understanding of this charge 6 gapped interface in the representation (2.8),

$$
\tilde{K}_{m, 3}=\left(\begin{array}{ccc}
2 m+1 & 2 m & 2 m \\
2 m & 2 m+1 & 2 m \\
2 m & 2 m & 2 m+1
\end{array}\right), \quad \tilde{q}_{m, 3}=(1,1,1),
$$

where the $K$ matrix and charge vectors resemble a trilayer FQH state, where each layer carries unit charge. The $\operatorname{SL}(3, \mathbb{Z})$ transformation to this new basis

$$
W_{3}=\left(\begin{array}{lll}
1 & 0 & 0 \\
1 & 1 & 0 \\
1 & 1 & 1
\end{array}\right)
$$

changes the null vector to

$$
\begin{aligned}
\tilde{\Lambda}_{1} & =\left[\left(W_{3}^{-1}\right)^{T} \oplus\left(W_{3}^{-1}\right)^{T}\right] \Lambda_{1} \\
& =(1,1,1,-1,-1,-1)^{T} .
\end{aligned}
$$


Then the interaction

$$
\begin{aligned}
U\left[\tilde{\Lambda}_{1}\right] & =\tilde{\lambda} \cos \left(\tilde{\Lambda}_{1} \tilde{\mathcal{K}}_{m, 3} \tilde{\Phi}\right) \\
& \sim \frac{\tilde{\lambda}}{2} \tilde{\psi}_{1 R} \tilde{\psi}_{2 R} \tilde{\psi}_{3 R} \tilde{\psi}_{1 L} \tilde{\psi}_{2 L} \tilde{\psi}_{3 L}+\text { H.c. }
\end{aligned}
$$

is manifestly a charge 6 operator involving pairing of three local fermions on each side of the interface. This interaction is a generalization of the charge 2 pairing at the interface of Laughlin states.

Similarly to the discussion of the first hierarchical state, we compare our set up with the theory of extrinsic defects associated with anyonic symmetries of the Abelian phase. In Ref. [13], the $\mathbb{Z}_{3}$ twisted defects associated with the layer permutations of the Abelian phase characterized by the $K$ matrix

$$
K_{m, \ell, \ell}=\left(\begin{array}{ccc}
m & \ell & \ell \\
\ell & m & \ell \\
\ell & \ell & m
\end{array}\right)
$$

where studied and it was shown that domain walls separating two distinct gapping charge conserving gap terms support parafermions with quantum dimension $d_{\mathbb{Z}_{3}}=|m-\ell|$. Comparing Eqs. (4.37) and (4.41) shows that that $\mathbb{Z}_{3}$ twist defect of the second hierarchical state is trivial, since $d_{\mathbb{Z}_{3}}=1$. Furthermore, it can be shown [53] that twist defects associated with charge conjugation anyonic symmetry are associated with Majorana fermions, in contrast with the $\mathbb{Z}_{2(6 m+1)}$ parafermions discussed here.

\section{Third hierarchical state $(m, 4)$}

The interface of the third hierarchical FQH state $(m, 4)$ with filling fraction $v_{m, 4}=\frac{4}{8 m+1}$ supports the low-energy mode fields $\left(\phi_{1}^{R}, \phi_{2}^{R}, \phi_{3}^{R}, \phi_{4}^{R}, \phi_{1}^{L}, \phi_{2}^{L}, \phi_{3}^{L}, \phi_{4}^{L}\right)$ where the Lagrangian (2.10) has

$$
\begin{aligned}
\mathcal{K}_{m, 4} & =K_{m, 4} \oplus\left(-K_{m, 4}\right), \quad Q_{m, 4}^{T}=\left(q_{m, 4}^{T},-q_{m, 4}^{T}\right), \\
K_{m, 4} & =\left(\begin{array}{cccc}
2 m+1 & -1 & 0 & 0 \\
-1 & 2 & -1 & 0 \\
0 & -1 & 2 & -1 \\
0 & 0 & -1 & 2
\end{array}\right), \quad q_{m, 4}^{T}=(1,0,0,0) .
\end{aligned}
$$

Charge neutral null vectors parametrizing the gap opening interactions (3.1a) read

$$
\begin{aligned}
& \Lambda_{1}^{(0)}=(1,0,0,0,1,0,0,0)^{T}, \\
& \Lambda_{2}^{(0)}=(0,1,0,0,0,1,0,0)^{T}, \\
& \Lambda_{3}^{(0)}=(0,0,1,0,0,0,1,0)^{T}, \\
& \Lambda_{4}^{(0)}=(0,0,0,1,0,0,0,1)^{T} .
\end{aligned}
$$

Carrying out an analysis similar to that discussed in Secs. IV B-IV D, we find that the null integer vectors of the charge nonconserving gapped interface are

$$
\Lambda_{i}=\left\{\begin{array}{ll}
(2,3,2,1,-2,0,0,0)^{T} & i=1 \\
\Lambda_{i}^{(0)} & i=2,3,4
\end{array} .\right.
$$

Notice that the interaction

$$
\begin{aligned}
U\left[\Lambda_{1}\right] & =\cos \left(\Lambda_{1} \mathcal{K}_{m, p} \Phi\right) \\
& \sim \psi_{1 R}^{2}\left(\psi_{2 R}^{3} \psi_{3 R}^{2} \psi_{4 R}\right) \psi_{1 L}^{2}+\text { H.c. }
\end{aligned}
$$

is a charge 4 cluster operator where $\psi_{1 R}$ and $\psi_{1 L}$ are charge 1 local operators with fermionic statistics and $\psi_{2 R}, \psi_{3 R}, \psi_{4 R}$ are charge zero local operators with bosonic statistics.

From Eqs. (4.44) and (3.5c), we get

$$
N_{m, 4}=\Lambda_{1}^{(0)} \mathcal{K}_{m, 4} \Lambda_{1}=(8 m+1),
$$

which establishes the presence of $\mathbb{Z}_{8 m+1}$ parafermions

$$
\alpha_{i} \alpha_{j}=e^{i \frac{2 \pi}{8 m+1} \operatorname{sgn}(i-j)} \alpha_{j} \alpha_{i}
$$

with quantum dimension

$$
d_{m, 4}=\sqrt{8 m+1}=\mathcal{D}_{m, 4} .
$$

This result shows that the parafermions in the third hierarchical state behave similarly to the first hierarchical state discussed in Sec. IV B. This nontrivial condensate is manifested in the expectation value of of the charge $\frac{4}{8 m+1}$ operator

$$
\left\langle\mathcal{O}_{\frac{4}{8 m+1}}\right\rangle \equiv\left\langle e^{i \frac{\Lambda_{1}^{(0)} \mathcal{K}_{m, 4} \Lambda_{1}}{8 m+1}}\right\rangle \neq 0 .
$$

So, for instance, the $v=4 / 9 \mathrm{FQH}$ state $(\mathrm{m}=1, \mathrm{p}=4)$ is seen to support $\mathbb{Z}_{9}$ parafermions along domain walls at its gapped edge. The charge 4 condensate is formed by a cluster of 9 quasiparticles with charge 4/9.

\section{HIERARCHY OF PARAFERMIONS: GENERAL CASE}

The properties of the parafermions zero modes stabilized on domain walls of the $p=1,2,3,4$ hierarchical states discussed in Sec. IV reveal a remarkable dependence on the parity of the index $p$, which labels the depth of the hierarchy. This dependence reflects an interplay between the bulk topological order, which gives rise to quasiparticle fractionalization, and the 1D SPT order that stabilizes Majorana zero modes in nontrivial topological superconductors. An appealing mechanism to account for such an even-odd dependence emerges when upon expressing the bulk topological order of the hierarchical state in the representation (2.8), where the $K$ matrix gives an interpretation of the bulk topological order as a series of $p$ Laughlin-type layers (as indicated in the diagonal odd integers $2 m+1$ ) coupled to each other by bosonic correlations (indicated by the off-diagonal even integers $2 m$ ). As such, the number of pairs of counter-propagating fermion modes in interfaces of hierarchical states $(m, p)$ and $(m, p+2)$ differ by 2 . In the limit, where these Laughlin FQH layers are decoupled [which would correspond to $K=$ $(2 m+1) \operatorname{diag}(1, \ldots, 1)]$, the even-odd effect associated with the stability of Majorana zero modes is a direct consequence of the $\mathbb{Z}_{2}$ stability (instability) associated with an odd (even) number of Majorana zero modes per domain. Remarkably, our analysis will show that this structure persists even when the layers are coupled, according to the $K$ matrix (2.8a).

One of the goals of this section is to show that this $\mathbb{Z}_{2}$ pattern indeed persists for all hierarchical states, whose bulk 
topological order are represented by Eq. (2.6) or, equivalently, Eq. (2.8). We will show that the interactions require breaking of charge conservation in such a way that the charge of the condensate depends on the hierarchical level $p$. Notably, while the primary Laughlin $(p=1)$ admit a charge 2 condensate that, in principle, can be induced by a weak-pairing mechanism (or by proximity to a superconductor), for generic states of the hierarchical sequence, the $\mathcal{Q}_{m, p}>2$ charge of the condensate signals that a non-BCS strong coupling mechanism is at play. This situation departs significantly from the stability of Majorana zero modes in superconducting wires [7] as well as in interfaces of Laughlin-type states [9-12].

An interesting property of the condensate is that it involves clustering of $2 m p+1$ quasiparticles of charge $v_{m, p}=\frac{p}{2 m p+1}$. This scenario of parafermions being stabilized by clustering of quasiparticles is analogous to clustering property of nonAbelian Read-Rezayi FQH states, [48] where electrons (or composite fermions) forming an order- $k$ cluster, give rise to an incompressible state that supports non-Abelian bulk excitations and chiral charge neutral $\mathbb{Z}_{k}$ parafermions on the boundary. (The special case $k=2$ corresponds to the MooreRead states with a chiral Majorana fermions at the boundary, which is a candidate topological order for the $v=5 / 2$ FQH.)

In Secs. V A and VB, we discuss, respectively, the $p=$ odd and $p=$ even hierarchical states in generality, where we shall provide explicit expressions for the null vectors and, consequently, the local interactions that gap the interface and give rise to parafermion zero modes localized on domain walls.

\section{A. Hierarchical states: $\boldsymbol{p}=$ odd integer}

Consider the interface between hierarchical FQH states with filling fraction

$$
v_{m, p}=\frac{p}{2 m p+1}, \quad m \in \mathbb{Z}_{+}, \quad p=1,3,5, \ldots
$$

As discussed in Sec. III, this interface admits a gapped region realized by the local charge neutral interactions (3.1). We now demonstrate that the interface formed by the states in Eq. (5.1) admits a set of local gap opening interactions that breaks $\mathrm{U}(1)$ charge conservation and represent a charge $\mathcal{Q}_{m, p}$ condensate where

$$
\mathcal{Q}_{m, p}=2 p, \quad m \in \mathbb{Z}_{+}, \quad p=1,3,5, \ldots
$$

In the representation (2.6), this condensate is realized by the local interactions

$$
U\left[\Lambda_{i}\right]=\cos \left(\Lambda_{i} \mathcal{K}_{m, p} \Phi\right), \quad i=1, \ldots, p,
$$

represented by the integer vectors

$$
\begin{gathered}
\Lambda_{1}=\left(\begin{array}{c}
v_{p} \\
-v_{p}
\end{array}\right), \quad v_{p}=\left(\begin{array}{c}
p \\
p-1 \\
\vdots \\
1
\end{array}\right), \\
\Lambda_{i}=\Lambda_{i}^{(0)}=\left(\begin{array}{c}
e_{i} \\
e_{i}
\end{array}\right), \quad i=2, \ldots, p,
\end{gathered}
$$

which satisfy the null condition

$$
\Lambda_{i} \mathcal{K}_{m, p} \Lambda_{j}, \quad i, j=1, \ldots, p .
$$

It follows immediately from Eq. (5.4) that the interaction corresponds to a condensate of charge $\mathcal{Q}\left[\Lambda_{1}\right]=\Lambda_{1} Q_{m, p}=$ $2 p$, in accordance with Eq. (5.2). (Note that for $2 \leqslant i \leqslant$ $p$ : $\mathcal{Q}\left[\Lambda_{i}\right]=\Lambda_{i} Q_{m, p}=0$.) Moreover, the nonzero minors of $\mathcal{M}[\{\Lambda\}]$ can be shown to form the set $\{ \pm(p-1), \pm p, \pm(p+$ $1)\}$ whose greatest common divisor is one, which shows that the integer vectors in Eq. (5.4) are primitive. Furthermore, to establish the null condition (5.5), we first realize that this condition is clearly satisfied for $i, j=2, \ldots, p$ as a consequence of Eq. (3.3). Then, the remaining nontrivial conditions we need to show are for $i=1$ and $j=1, \ldots, p$. To establish this result, all we need is the identity

$$
K_{m, p} v_{p}=\left(\begin{array}{c}
2 m p+1 \\
0 \\
\vdots \\
0
\end{array}\right)=(2 m p+1) e_{1}
$$

where $v_{p}$ is $p$-dimensional integer vector defined in Eq. (5.4). To demonstrate this result, let $K_{m, p} v_{p}=\sum_{k=1}^{p} a_{k} e_{k}$. Except for the first and last rows, the remaining rows of $K_{m, p}$ are formed by consecutive entries $-1,2,1$ and the remaining ones equal to zero. The first row has $\left(K_{m, p}\right)_{11}=2 m+1$ and $\left(K_{m, p}\right)_{12}=-1$. The last row has $\left(K_{m, p}\right)_{p, p-1}=-1$ and $\left(K_{m, p}\right)_{p, p}=2$. Putting all together,

$$
\begin{aligned}
a_{1} & =p(2 m+1)+(-1) \times(p-1)=2 m p+1, \\
a_{k} & =(-1) \times(k+1)+2 \times(k)+(-1) \times(k-1)=0, \\
2 & \leqslant k \leqslant p-1, \\
a_{p} & =(-1) \times(2)+2 \times(1)=0,
\end{aligned}
$$

which proves Eq. (5.6). Finally, by taking into account the orthonormal basis vectors $e_{i}$, it is straightforward to verify the null condition (5.5).

The form of the charge $2 p$ interaction, which follows directly from Eq. (5.6), is

$$
U\left[\Lambda_{1}\right]=\cos \left(\Lambda_{1} \mathcal{K}_{m, p} \Phi\right)=\cos \left[(2 m p+1)\left(\phi_{1}^{R}+\phi_{1}^{L}\right)\right] .
$$

In the basis given by Eq. (2.8) the integer vectors $\Lambda_{1}$ transforms to

$$
\tilde{\Lambda}_{1}=\left(W^{-1}\right)^{T} \Lambda_{1}=(1,1, \ldots, 1,-1,-1, \ldots,-1)^{T} .
$$

The meaning of the charge $2 p$ interaction in this representation is manifestly given by

$$
\begin{aligned}
U\left[\tilde{\Lambda}_{1}\right] & =\cos \left(\tilde{\Lambda}_{1} \tilde{\mathcal{K}}_{m, p} \tilde{\Phi}\right) \\
& \sim\left(\tilde{\psi}_{1}^{L} \tilde{\psi}_{1}^{R}\right) \ldots\left(\tilde{\psi}_{p}^{L} \tilde{\psi}_{p}^{R}\right)+\text { H.c. }
\end{aligned}
$$

which represents the cluster of $2 p$ fermions, each one carrying charge $q=1$. Equation (5.10) generalizes, to every odd value of $p$, the charge 6 interaction that gaps the interface of the hierarchical state $(m, 3)$ depicted in Fig. 4 .

Finally, the quantum dimension of the parafermion localized at the domain wall between the segments of the interface 


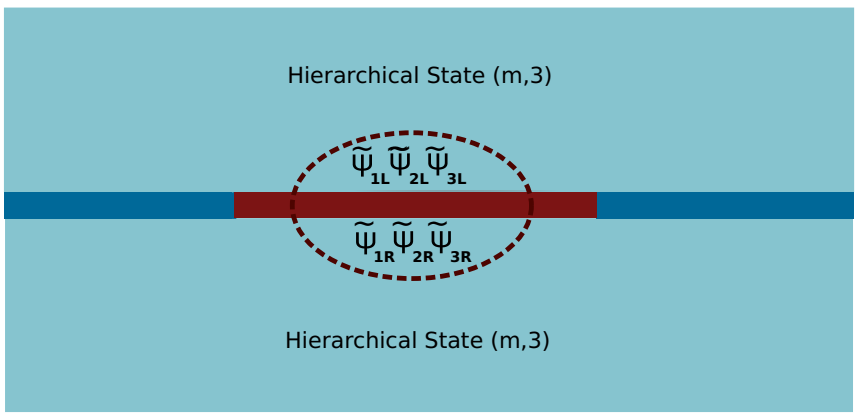

FIG. 4. Interface between the second hierarchical FQH state $(m, 3)$. The red segment represents the interface gapped by the charge 6 interaction (4.40).

that are gapped by interactions (3.1a) and (5.3) follows from

$$
\begin{aligned}
N_{m, p}=\Lambda_{1}^{(0)} \mathcal{K}_{m, p} \Lambda_{1} & =\left(\begin{array}{ll}
e_{1}^{T} & e_{1}^{T}
\end{array}\right)\left(\begin{array}{l}
(2 m p+1) e_{1} \\
(2 m p+1) e_{1}
\end{array}\right) \\
& =2(2 m p+1),
\end{aligned}
$$

which establishes the existence of parafermions of quantum dimension

$$
d_{m, p}=\sqrt{2} \times \sqrt{2 m p+1},
$$

at the interface of hierarchical states filling fraction $v_{m, p}=$ $\frac{p}{2 m p+1}, m \in \mathbb{Z}_{+}, p=1,3,5, \ldots$

\section{B. Hierarchical states: $p=$ even integer}

Consider the interface between hierarchical FQH states with filling fraction

$$
v_{m, p}=\frac{p}{2 m p+1}, \quad m \in \mathbb{Z}_{+}, \quad p=2,4,6, \ldots
$$

As discussed in Sec. III, this interface admits a gapped region realized by the local charge neutral interactions (3.1). We now prove that the interface formed by the states in Eq. (5.13) can be gapped by local interactions that break charge conservation symmetry and give rise to a condensate of charge

$$
\mathcal{Q}_{m, p}=p, \quad m \in \mathbb{Z}_{+}, \quad p=2,4,6, \ldots
$$

In the representation (2.6), this charge $p$ condensate is realized by the local interactions

$$
U\left[\Lambda_{i}\right]=\cos \left(\Lambda_{i} \mathcal{K}_{m, p} \Phi\right),
$$

where the integer vectors read

$$
\begin{aligned}
& \Lambda_{i} \\
& = \begin{cases}\left(\frac{p}{2}, p-1, p-2, \ldots, 1,-\frac{p}{2}, 0,0, \ldots, 0\right)^{T} & i=1 \\
\Lambda_{i}^{(0)} & i=2, \ldots, p\end{cases}
\end{aligned}
$$

and satisfy the null condition

$$
\Lambda_{i} \mathcal{K}_{m, p} \Lambda_{j}=0, \quad \forall i, \quad j=1, \ldots, p .
$$

With the null vectors (5.16) we directly find that this corresponds to a condensate of charge $\mathcal{Q}\left[\Lambda_{1}\right]=\Lambda_{1} Q_{m, p}=p$, as given by Eq. (5.14). (Notice that for $2 \leqslant i \leqslant p: \mathcal{Q}\left[\Lambda_{i}\right]=$ $\Lambda_{i} Q_{m, p}=0$.) Moreover, from the explicit form of the integer vector $\Lambda_{1}$ in Eq. (5.16), one verifies that the charge $p$ interaction

$$
\begin{aligned}
U\left[\Lambda_{1}\right] & =\cos \left(\Lambda_{1} \mathcal{K}_{m, p} \Phi\right) \\
& \sim \psi_{1 R}^{p / 2}\left(\psi_{2 R}^{p-1} \psi_{3 R}^{p-2} \ldots \psi_{(p-1) R}^{2} \psi_{p R}\right) \psi_{1 L}^{p / 2}+\text { H.c. }
\end{aligned}
$$

represents a cluster operator where $\psi_{1 R}$ and $\psi_{1 L}$ are charge 1 local operators with fermionic statistics and $\psi_{2 R}, \ldots, \psi_{p R}$ are charge zero local operators with bosonic statistics.

We now demonstrate the validity of Eq. (5.17). Since $\left\{\Lambda_{i}^{(0)}\right\}, i=2, \ldots, p$ forms, by construction, a subset of null vectors, the only nontrivial relations left to be verified are $\Lambda_{i} \mathcal{K}_{m, p} \Lambda_{1}=0$ for $i=1, \ldots, p$. In order to establish these conditions, we directly calculate

$$
\mathcal{K}_{m, p} \Lambda_{1}=\left(\begin{array}{c}
\frac{p}{2}(2 m-1)+1 \\
\frac{p}{2} \\
0 \\
\vdots \\
0 \\
0 \\
\frac{p}{2}(2 m+1) \\
-\frac{p}{2} \\
0 \\
\vdots \\
0
\end{array}\right),
$$

leading to

$$
\begin{aligned}
\Lambda_{1} \mathcal{K}_{m, p} \Lambda_{1}= & \frac{p}{2}\left[\frac{p}{2}(2 m-1)+1\right] \\
& +(p-1) \frac{p}{2}-\frac{p}{2}\left[\frac{p}{2}(2 m+1)\right]=0, \\
\Lambda_{2} \mathcal{K}_{m, p} \Lambda_{1}= & \frac{p}{2}-\frac{p}{2}=0 \\
\Lambda_{i} \mathcal{K}_{m, p} \Lambda_{1}= & 0, \quad i=3, \ldots, p
\end{aligned}
$$

where the last equation is a consequence of $e_{i}^{T} \cdot e_{1,2}=0$ for $i=3, \ldots, p$. This then establishes the null condition (5.17).

Finally, the quantum dimension of parafermion localized at the domain wall between the segments of the interface that are gapped by interactions (3.1a) and (5.15) follows from

$$
N_{m, p}=\Lambda_{1}^{(0)} \mathcal{K}_{m, p} \Lambda_{1}=2 m p+1,
$$

which shows establishes the existence of parafermions with quantum dimension

$$
d_{m, p}=\sqrt{2 m p+1}
$$

at the interface with filling fraction $v_{m, p}=\frac{p}{2 m p+1}$, with $m \in \mathbb{Z}_{+}$and $p=2,4,6, \ldots$

We have then explicitly demonstrated, in Secs. VA and V B, the existence of a local charge condensate, Eq. (1.2), 
that stabilizes non-Abelian parafermions with quantum dimensions given by Eq. (1.3) and depicted in Fig. 1.

\section{SUMMARY AND OUTLOOK}

In this work, we have established a correspondence between the sequence of Abelian hierarchical $\mathrm{FQH}$ states in the first Landau level and a class of extrinsic non-Abelian zero modes localized on domain walls that separate charge neutral and $\mathrm{U}(1)$ symmetry broken gapped segments of the interfaces. Our analysis of the low-energy properties of the bulk hierarchical state employed the hydrodynamical Chern-Simons theory by which the FQH system with Hall conductance $\sigma_{x y}(m, p)=\frac{e^{2}}{h} \frac{p}{2 m p+1}$ is represented in terms of a $p$-component $\mathrm{U}(1)$ Chern-Simons gauge theory parametrized by an integer valued $K$ matrix [36-40]. The edge of such hierarchical state, in turn, supports $p$ chiral low-energy modes described by a $p$-component chiral boson field whose commutation relations are determined by the $K$ matrix. As such, we have studied gap opening processes in a homogeneous interface with $p$ pairs of counter-propagating modes, as depicted in Fig. 2. Gapping these modes at the interface is realized by $p$ local sine-Gordon type operators.

Through a detailed examination of the locality and frustration free conditions of sine-Gordon operators on the homogeneous interface, we have found that the hierarchical states admit $U(1)$ symmetry breaking interactions that give rise to a condensate whose charge is a function of the hierarchical index $p$, as per Eq. (1.2). Therefore our results show that a charge 2 condensate only occurs for $p=1$ and 2, i.e., for the primary Laughlin states with filling fraction $\frac{1}{2 m+1}$ and the first hierarchical states with filling fraction $\frac{2}{4 m+1}$, for integer $m>1$. (We note that charge 2 condensates formed the basis of earlier studies of parafermions in interfaces and trenches of Laughlin states [9-12] and the particle-hole conjugate of the $v=1 / 3$ Laughlin state at filling $v=2 / 3$ [15].) For the general $p>2$ case investigated in this work, on the other hand, we have found that the local U(1) symmetry breaking interactions involve a nonconventional charge clustering mechanism whereby more than two electrons are glued together. Our findings then open the interesting possibility of exploring these gapped interfaces as a basis for constructing families of unconventional U(1) symmetry broken phases in $2 \mathrm{D}$ by promoting the interfaces to a "wire network", in the spirit of Ref. [54].

One of the main results of this work was establishing that the properties of parafermions zero modes stem from the existence of a cluster state of charge given by Eq. (1.2), which translates into a cluster of fractionalized quasiparticles of charge $p /(2 m p+1)$. This state bears a striking resemblance with the Read-Rezayi FQH states that represent non-Abelian $\mathrm{FQH}$ states where electrons form cluster states. In fact, this correspondence has been explored in Ref. [15], where it was shown that superconducting islands in the $v=2 / 3 \mathrm{FQH}$ state harbor $\mathbb{Z}_{3}$ parafermions that are closely related to the neutral parafermion excitations of the $\mathbb{Z}_{3}$ Read-Rezayi FQH, whose ground-state wavefunction encodes clustering of three electrons. From this perspective, the results obtained here for the entire sequence of Abelian hierarchical $\mathrm{FQH}$ states establish a rich connection between two distinct families of Abelian and non-Abelian topological orders, and suggest, in particular, a route to describe the hierarchy of non-Abelian phases [55] via the deconfinement of extrinsic parafermion zero modes in corresponding Abelian phases of matter, which is an important topic worth of further investigation. Furthermore, since the parafermions in the setting considered here do not manifest any direct relationship with anyonic symmetries, the deconfinement of these non-Abelian defects may require a theoretical treatment that differs from those of Refs. [56,57], which dealt with the deconfinement of twist defects related to symmetries of the anyon group.

We have found an appealing dependence of the charge condensate and the quantum dimension of parafermions on the parity of the level hierarchy $p$, as shown in Fig. 1. We have argued that the even-odd dependence on $p$ is indicative of the $\mathbb{Z}_{2}$ stability of Majorana zero modes in 1D topological superconductors, where the parity of $p$ matches the parity of Majorana zero models per domain, according to the quantum dimension (1.3). This parity dependence is naturally accounted for in the multilayer representation of the $K$ matrix of the hierarchical states, despite the fact that each hierarchical state we studied is understood to be realized in a monolayer system in the lowest Landau level. According to this result, the quantum dimension of the parafermions depends both on the bulk Abelian topological order via the total quantum dimension $\mathcal{D}_{m, p}$ of the hierarchical Abelian bulk phase, as well as on the Majorana modes stabilized by conservation of fermion parity in 1D gapped fermionic phases of matter [58-60].

We close by pointing to a relation between the nonzero charge condensate at the interface of hierarchical $\mathrm{FQH}$ states and the entanglement entropy associated with an entanglement cut across the interface [61-64]. In that regard, it is possible to show that the interactions giving rise to the charge condensate in the hierarchical states are invariant under a global $\mathbb{Z}_{k} \times \mathbb{Z}_{k}$ symmetry [where $k=p(k=p / 2)$ for odd (even) values of $p$ ], which correspond to transformations on the local operators having support on each side of the interface. It was shown in Ref. [65] that the existence of local gap opening interactions possessing such discrete symmetry gives rise to a nontrivial $\ln (k)$ correction to the bulk universal value of the topological entanglement entropy, which, in nonchiral bulk Abelian phases, characterize the onset of a 1D gapped SPT chain along the interface. (See also Ref. [66] for an early discussion of entanglement corrections in 2D Abelian phases of matter and Ref. [67] for a relationship between such entanglement corrections and string order parameters.) We stress, however, that hierarchical states studied here are chiral phases, which implies that the parafermion zero modes are not protected by the emergent $\mathbb{Z}_{k} \times \mathbb{Z}_{k}$ symmetry of the local interactions that stabilize the condensate. Nevertheless, we note that the local interactions we have discussed here by no means exhaust the possible classes of gapped interfaces that can be formed in hierarchical states. It is then an important open question whether such hierarchical interfaces can support also genuine 1D SPT phases of matter protected by other classes of discrete symmetries, as recently discussed in Ref. [65].

In summary, leveraging on the anyon condensation mechanism that gives rises to the hierarchical sequence of Abelian 
$\mathrm{FQH}$ states, we have discovered a remarkably rich sequence of non-Abelian parafermions that are stabilized by clustered states of electrons and quasiparticles on their interfaces. Our study opens an exciting possibility to gain a deeper understanding of the structure of $2 \mathrm{D}$ non-Abelian states by exploring more familiar and well understood Abelian phases of matter.

\section{ACKNOWLEDGMENTS}

I wish to thank Jason Alicea for an insightful discussion and Paul Fendley for a feedback on the manuscript. L.H.S. is supported by a faculty startup at Emory University. This work was performed in part at the Aspen Center for Physics, which is supported by National Science Foundation Grant No. PHY-1607611.
[1] X.-G. Wen, Quantum Field Theory of Many-Body Systems (Oxford University Press, New York, 2004).

[2] C. Nayak, S. H. Simon, A. Stern, M. Freedman, and S. Das Sarma, Non-Abelian anyons and topological quantum computation, Rev. Mod. Phys. 80, 1083 (2008).

[3] G. Moore and N. Read, Non-Abelions in the fractional quantum Hall effect, Nucl. Phys. B 360, 362 (1991).

[4] A. Kitaev, Anyons in an exactly solved model and beyond, Ann. Phys. 321, 2 (2006).

[5] M. Banerjee, M. Heiblum, V. Umansky, D. E. Feldman, Y. Oreg, and A. Stern, Observation of half-integer thermal Hall conductance, Nature (London) 559, 205 (2018).

[6] Y. Kasahara, T. Ohnishi, Y. Mizukami, O. Tanaka, S. Ma, K. Sugii, N. Kurita, H. Tanaka, J. Nasu, Y. Motome, T. Shibauchi, and Y. Matsuda, Majorana quantization and half-integer thermal quantum Hall effect in a Kitaev spin liquid, Nature (London) 559, 227 (2018).

[7] A. Yu. Kitaev, Unpaired Majorana fermions in quantum wires, Phys. Usp. (Suppl.) 44, 131 (2001).

[8] M. Barkeshli and X.-L. Qi, Topological Nematic States and Non-Abelian Lattice Dislocations, Phys. Rev. X 2, 031013 (2012).

[9] N. H. Lindner, E. Berg, G. Refael, and A. Stern, Fractionalizing Majorana Fermions: Non-Abelian Statistics on the Edges of Abelian Quantum Hall States, Phys. Rev. X 2, 041002 (2012).

[10] D. J. Clarke, J. Alicea, and K. Shtengel, Exotic non-Abelian anyons from conventional fractional quantum Hall states, Nat. Commun. 4, 1348 (2013).

[11] M. Cheng, Superconducting proximity effect on the edge of fractional topological insulators, Phys. Rev. B 86, 195126 (2012).

[12] A. Vaezi, Fractional topological superconductor with fractionalized Majorana fermions, Phys. Rev. B 87, 035132 (2013).

[13] M. Barkeshli, C.-M. Jian, and X.-L. Qi, Twist defects and projective non-Abelian braiding statistics, Phys. Rev. B 87, 045130 (2013).

[14] M. Barkeshli, C.-M. Jian, and X.-L. Qi, Classification of topological defects in Abelian topological states, Phys. Rev. B 88, 241103(R) (2013).

[15] R. S. K. Mong, D. J. Clarke, J. Alicea, N. H. Lindner, P. Fendley, C. Nayak, Y. Oreg, A. Stern, E. Berg, K. Shtengel, and M. P. A. Fisher, Universal Topological Quantum Computation from a Superconductor-Abelian Quantum Hall Heterostructure, Phys. Rev. X 4, 011036 (2014).

[16] M. N. Khan, J. C. Y. Teo, and T. L. Hughes, Anyonic symmetries and topological defects in abelian topological phases: An application to the $A D E$ classification, Phys. Rev. B 90, 235149 (2014).
[17] L. H. Santos and T. L. Hughes, Parafermionic Wires at the Interface of Chiral Topological States, Phys. Rev. Lett. 118, 136801 (2017).

[18] V. Ostrik, Module categories over the Drinfeld double of a finite group, Int. Math. Res. Not. 2003, 1507 (2003).

[19] M. Barkeshli and X.-G. Wen, $u(1) \times u(1) \rtimes Z_{2}$ Chern-Simons theory and $Z_{4}$ parafermion fractional quantum Hall states, Phys. Rev. B 81, 045323 (2010).

[20] H. Bombin, Topological Order with a Twist: Ising Anyons from an Abelian Model, Phys. Rev. Lett. 105, 030403 (2010).

[21] S. Beigi, P. W. Shor, and D. Whalen, The quantum double model with boundary: Condensations and symmetries, Commun. Math. Phys. 306, 663 (2011).

[22] P. Fendley, Parafermionic edge zero modes in Zn-invariant spin chains, J. Stat. Mech. (2012) P11020.

[23] E. Fradkin and L. P. Kadanoff, Disorder variables and parafermions in two-dimensional statistical mechanics, Nucl. Phys. B 170, 1 (1980).

[24] V. A. Fateev and A. B. Zamolodchikov, Parafermionic currents in the two-dimensional conformal quantum field theory and selfdual critical points in Z(n) invariant statistical systems, Sov. Phys. JETP 62, 215 (1985) [Zh. Eksp. Teor. Fiz. 89, 380 (1985)].

[25] J. Alicea and P. Fendley, Topological phases with parafermions: Theory and blueprints, Annu. Rev. Condens. Matter Phys. 7, 119 (2016).

[26] Disregarding constraints from the Hilbert space.

[27] N. Read and D. Green, Paired states of fermions in two dimensions with breaking of parity and time-reversal symmetries and the fractional quantum Hall effect, Phys. Rev. B 61, 10267 (2000).

[28] D. A. Ivanov, Non-Abelian Statistics of Half-Quantum Vortices in $p$-Wave Superconductors, Phys. Rev. Lett. 86, 268 (2001).

[29] R. E. Prange and S. M. Girvin, The Quantum Hall Effect (Springer-Verlag, Heidelberg, 1987).

[30] R. B. Laughlin, Anomalous Quantum Hall Effect: An Incompressible Quantum Fluid with Fractionally Charged Excitations, Phys. Rev. Lett. 50, 1395 (1983).

[31] J. K. Jain, Composite-Fermion Approach for the Fractional Quantum Hall Effect, Phys. Rev. Lett. 63, 199 (1989).

[32] F. D. M. Haldane, Fractional Quantization of the Hall Effect: A Hierarchy of Incompressible Quantum Fluid States, Phys. Rev. Lett. 51, 605 (1983).

[33] B. I. Halperin, Statistics of Quasiparticles and the Hierarchy of Fractional Quantized Hall States, Phys. Rev. Lett. 52, 1583 (1984).

[34] A. López and E. Fradkin, Fractional quantum Hall effect and Chern-Simons gauge theories, Phys. Rev. B 44, 5246 (1991). 
[35] W. Pan, H. L. Stormer, D. C. Tsui, L. N. Pfeiffer, K. W. Baldwin, and K. W. West, Fractional Quantum Hall Effect of Composite Fermions, Phys. Rev. Lett. 90, 016801 (2003).

[36] B. Blok and X. G. Wen, Effective theories of the fractional quantum Hall effect at generic filling fractions, Phys. Rev. B 42, 8133 (1990).

[37] N. Read, Excitation Structure of the Hierarchy Scheme in the Fractional Quantum Hall Effect, Phys. Rev. Lett. 65, 1502 (1990).

[38] J. Fröhlich and A. Zee, Large scale physics of the quantum Hall fluid, Nucl. Phys. B 364, 517 (1991).

[39] X. G. Wen and A. Zee, Classification of Abelian quantum Hall states and matrix formulation of topological fluids, Phys. Rev. B 46, 2290 (1992).

[40] J. Fröhlich and E. Thiran, Integral quadratic forms, Kac-Moody algebras, and fractional quantum Hall effect. Anade-o classification, J. Stat. Phys. 76, 209 (1994).

[41] L. Santos, T. Neupert, S. Ryu, C. Chamon, and C. Mudry, Time-reversal symmetric hierarchy of fractional incompressible liquids, Phys. Rev. B 84, 165138 (2011).

[42] M. Levin and A. Stern, Classification and analysis of two-dimensional Abelian fractional topological insulators, Phys. Rev. B 86, 115131 (2012).

[43] X.-G. Wen, Topological orders and edge excitations in fractional quantum Hall states, Adv. Phys. 44, 405 (1995).

[44] F. D. M. Haldane, Stability of Chiral Luttinger Liquids and Abelian Quantum Hall States, Phys. Rev. Lett. 74, 2090 (1995).

[45] J. Bardeen, L. N. Cooper, and J. R. Schrieffer, Theory of superconductivity, Phys. Rev. 108, 1175 (1957).

[46] A. Kitaev and J. Preskill, Topological Entanglement Entropy, Phys. Rev. Lett. 96, 110404 (2006).

[47] M. Levin and X.-G. Wen, Detecting Topological Order in a Ground State Wave Function, Phys. Rev. Lett. 96, 110405 (2006).

[48] N. Read and E. Rezayi, Beyond paired quantum Hall states: Parafermions and incompressible states in the first excited Landau level, Phys. Rev. B 59, 8084 (1999).

[49] E. M. Stoudenmire, D. J. Clarke, R. S. K. Mong, and J. Alicea, Assembling Fibonacci anyons from a $\mathbb{Z}_{3}$ parafermion lattice model, Phys. Rev. B 91, 235112 (2015).

[50] F. Wilczek, Magnetic Flux, Angular Momentum, and Statistics, Phys. Rev. Lett. 48, 1144 (1982).

[51] F. Amet, C. T. Ke, I. V. Borzenets, J. Wang, K. Watanabe, T. Taniguchi, R. S. Deacon, M. Yamamoto, Y. Bomze, S. Tarucha, and G. Finkelstein, Supercurrent in the quantum Hall regime, Science 352, 966 (2016).

[52] G.-H. Lee, K.-F. Huang, D. K. Efetov, Di S. Wei, S. Hart, T. Taniguchi, K. Watanabe, A. Yacoby, and P. Kim, Inducing superconducting correlation in quantum Hall edge states, Nat. Phys. 13, 693 (2017).

[53] Let there be a segment of the interface gapped by local charge neutral interactions parametrized by the null vectors $\left\{\Lambda_{i}^{(0)}=\right.$ $\left.\left(e_{i}, e_{i}\right) \mid i=1, \ldots, p\right\}$ and another one gapped by the charge 2 interactions with $\left\{\Lambda_{i}^{(2)}=\left(e_{i},-e_{i}\right) \mid i=1, \ldots, p\right\}$. Analysis of the commutation relations of the operators similar to Eq. (3.5) shows that the quantum dimension of the zero mode is given the greatest common divisor of the set of integer numbers $\left\{\Lambda_{i}^{(0)} \mathcal{K}_{m, p} \Lambda_{j}^{(2)} \mid i, j=1, \ldots, p\right\}$. From the structure of the $\mathrm{K}$ matrix of the hierarchical state, we find that the greatest common divisor of this set is 2, which corresponds to Majorana zero modes at the domain wall.

[54] J. C. Y. Teo and C. L. Kane, From Luttinger liquid to non-Abelian quantum Hall states, Phys. Rev. B 89, 085101 (2014).

[55] T. Lan and X.-G. Wen, Hierarchy Construction and NonAbelian Families of Generic Topological Orders, Phys. Rev. Lett. 119, 040403 (2017).

[56] M. Barkeshli, P. Bonderson, M. Cheng, and Z. Wang, Symmetry fractionalization, defects, and gauging of topological phases, Phys. Rev. B 100, 115147 (2019).

[57] J. C. Y. Teo, T. L. Hughes, and E. Fradkin, Theory of twist liquids: Gauging an anyonic symmetry, Ann. Phys. 360, 349 (2015).

[58] X.-L. Qi, T. L. Hughes, and S.-C. Zhang, Topological field theory of time-reversal invariant insulators, Phys. Rev. B 78, 195424 (2008).

[59] A. P. Schnyder, S. Ryu, A. Furusaki, and A. W. W. Ludwig, Classification of topological insulators and superconductors, in Advances in Theoretical Physics: Landau Memorial Conference, edited by V. Lebedev and M. Feigel'man, AIP Conf. Proc. No. 1134 (AIP, New York, 2009), p. 10.

[60] A. Kitaev, Periodic table for topological insulators and superconductors, in Advances in Theoretical Physics: Landau Memorial Conference, edited by V. Lebedev and M. Feigel'man, AIP Conf. Proc. No. 1134 (AIP, New York, 2009), p. 22.

[61] X.-L. Qi, H. Katsura, and A. W. W. Ludwig, General Relationship Between the Entanglement Spectrum and the Edge State Spectrum of Topological Quantum States, Phys. Rev. Lett. 108, 196402 (2012).

[62] R. Lundgren, Y. Fuji, S. Furukawa, and M. Oshikawa, Entanglement spectra between coupled Tomonaga-Luttinger liquids: Applications to ladder systems and topological phases, Phys. Rev. B 88, 245137 (2013).

[63] X. Chen and E. Fradkin, Quantum entanglement and thermal reduced density matrices in fermion and spin systems on ladders, J. Stat. Mech. (2013) P08013.

[64] S. Furukawa and Y. B. Kim, Entanglement entropy between two coupled Tomonaga-Luttinger liquids, Phys. Rev. B 83, 085112 (2011).

[65] L. H. Santos, J. Cano, M. Mulligan, and T. L. Hughes, Symmetry-protected topological interfaces and entanglement sequences, Phys. Rev. B 98, 075131 (2018).

[66] J. Cano, T. L. Hughes, and M. Mulligan, Interactions along an entanglement cut in $2+1 \mathrm{D}$ Abelian topological phases, Phys. Rev. B 92, 075104 (2015).

[67] D. J. Williamson, A. Dua, and M. Cheng, Spurious Topological Entanglement Entropy from Subsystem Symmetries, Phys. Rev. Lett. 122, 140506 (2019). 